\title{
QUANTITATIVE OPTICAL IMAGING OF VASCULAR STRUCTURE AND FUNCTION IN A MODEL OF PERIPHERAL ARTERIAL DISEASE
}

\author{
By
}

Kristin Marie Poole

Thesis

Submitted to the Faculty of the

School of Engineering of Vanderbilt University

in partial fulfillment of the requirements

for the degree of

\section{MASTER OF SCIENCE \\ in \\ Biomedical Engineering}

August, 2012

Nashville, Tennessee

Approved:

Professor Craig L. Duvall

Professor Melissa C. Skala 


\section{ACKNOWLEDGEMENTS}

This work would not have been possible without the financial support provided by Vanderbilt University Start-Up funding for Professors Melissa C. Skala and Craig L. Duvall, the IBM Graduate Fellowship Award, the National Science Foundation Graduate Research Fellowship, and the Vanderbilt Discovery Grant. Experiments were performed in part through the use of equipment in the Vanderbilt University Institute of Imaging Science and in the labs of Dr. Anita Mahadevan-Jansen and Dr. Lillian Nanney. I would like to thank my advisors, Dr. Craig Duvall and Dr. Melissa Skala, for their guidance, patience, and combined expertise in vascular disease and optics as I pursued this work. I would also like to thank Dr. Chetan Patil, Jason Tucker-Schwartz and Andrew Fontanella for answering my many questions about OCT and developing code for image processing, and Alex Walsh, Wesley Sit and Alec LaFontant for their assistance during my experiments. Finally, I would like to thank my parents Shane and Cheryl, my sisters Katelyn and Kara, and Brian O'Grady for their unwavering support of all of my academic and personal endeavors. 


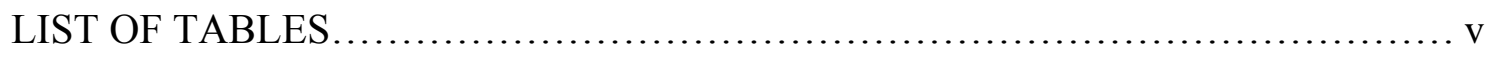

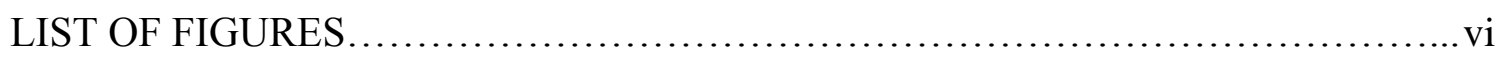

\section{CHAPTER}

1. INTRODUCTION .......................................................... 1

Overview of Peripheral Arterial Disease................................................. 1

Introduction.......................................................... 1

Risk Factors and Diagnosis............................................ 2

Prognosis and Treatment............................................. 3

Preclinical Models and Methods............................................. 8

Introduction....................................................... 8

Current Techniques for Evaluating Vascular Recovery..................... 9

Motivation............................................................ 10

Hyperspectral Imaging........................................................ 11

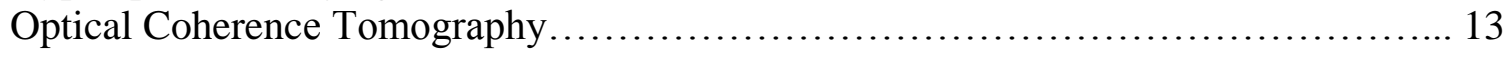

2. QUANTITATIVE, INTRAVITAL OPTICAL IMAGING OF VASCULAR STRUCTURE AND FUNCTION IN A MODEL OF PERIPHERAL ARTERIAL

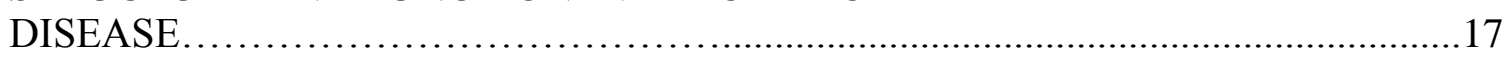

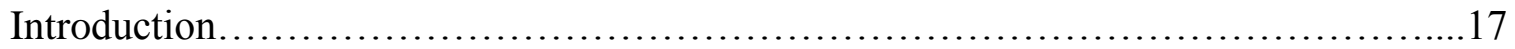

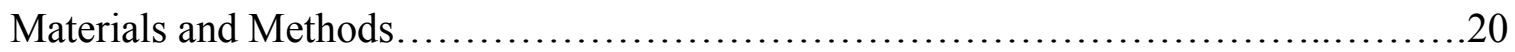

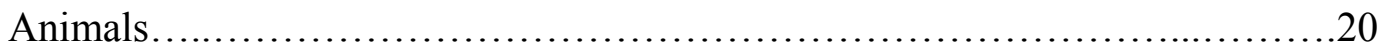

Hind Limb Ischemia Model..............................................20

Hyperspectral Imaging................................................21

Laser Doppler Perfusion Imaging..........................................24

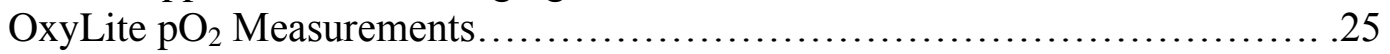

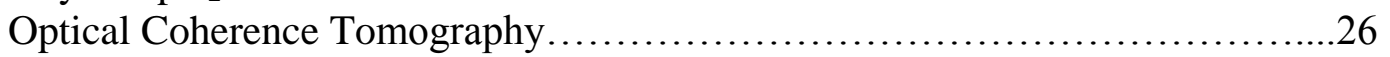

Statistical Analysis....................................................29

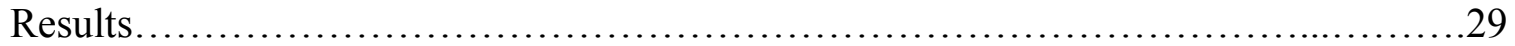

Perfusion Measurements with LDPI........................................29

Tissue Oxygen Tension in the Hind Feet................................... 31

Measurement of Hemoglobin Oxygen Saturation.............................. 32

Doppler Optical Coherence Tomography Imaging.............................38

Discussion................................................................. 42

Limitations of Standard Techniques for Hind Limb Ischemia Studies...........42

Hemoglobin Oxygen Saturation in Hind Limb Ischemia........................44

OCT Imaging of Vessel Function and Morphology..........................47

Conclusions and Perspectives...........................................49

3. CONCLUSIONS AND FUTURE WORK .................................... 51 
Quantitative Endpoints for Optical Imaging Methods................................. 52

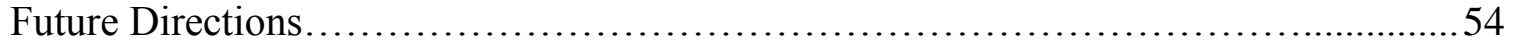

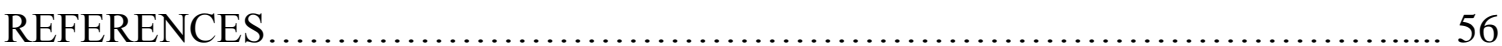




\section{LIST OF TABLES}

Table

Page

1.1 Gene Therapy in Peripheral Arterial Disease: Bioactivity Data ...................

3.1 Endpoints to be Derived from Speckle Variance and Doppler OCT Data .........53 


\section{LIST OF FIGURES}

$\begin{array}{lll}\text { Figure } & \text { Page }\end{array}$

1.1 Concept of therapeutic angiogenesis using DNA of growth factors ........... 5

1.2 Absorbance spectra of oxy- and deoxyhemoglobin ........................... 12

2.1 Schematic of hyperspectral system for diffuse reflectance imaging $\ldots \ldots \ldots \ldots \ldots .22$

2.2 Validation of hemoglobin oxygen saturation measurement ....................23

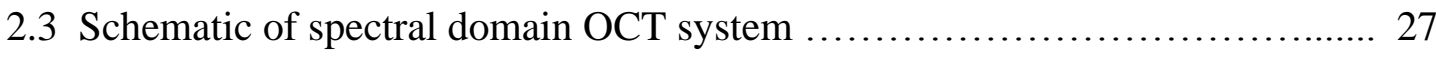

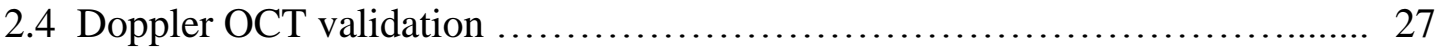

2.5 Measurements with LDPI and the OxyLite tissue oxygenation sensor .......... 30

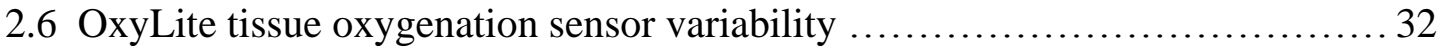

2.7 Hemoglobin oxygen saturation measured with hyperspectral imaging .......... 34

2.8 Trends in oxyhemoglobin and deoxyhemoglobin content after surgery ........... 36

2.9 Hemoglobin oxygen saturation correlates with perfusion and tissue $\mathrm{pO}_{2} \ldots \ldots \ldots 37$

2.10 Hyperspectral imaging of hemoglobin oxygen saturation is less variable .......38

2.11 Doppler OCT reveals changes in flow through the femoral artery and vein .... 40

2.12 Representative maximum intensity projections of Doppler volume data ...... 41

3.1 Doppler OCT of femoral artery and vein in a control hind limb .............. 53 


\title{
CHAPTER 1
}

\section{INTRODUCTION}

\section{Overview of Peripheral Arterial Disease}

\author{
Introduction
}

As many as 8 million American adults are affected by peripheral arterial disease (PAD) which is associated with significant morbidity and mortality (1). PAD is an occlusive arterial disease affecting non-coronary arteries, and it is most often used to describe disease of the arteries supplying the lower extremities. Symptoms include intermittent claudication, non-healing ulcers, ischemic rest pain and critical limb ischemia (2). PAD is also associated with increased risk of myocardial infarction and stroke $(3,4)$. Therapy for PAD patients consists of modification of risk factors, exercise, pharmacotherapy, and surgical revascularization (5). However, some patients may have prior revascularization procedures or diffuse atherosclerosis and therefore be poor candidates for surgical intervention (6). Therapeutic angiogenesis has been investigated as an adjunct approach to managing PAD through delivery of angiogenic growth factors such as vascular endothelial growth factor (VEGF) in recombinant protein or gene delivery form. This strategy aims to increase blood flow to ischemic tissue by inducing formation of a collateral vessel network $(6,7)$. Some of the early clinical trials have shown mildly promising results, but quantitative analysis of the efficacy of new therapeutics is lacking (6). 


\section{$\underline{\text { Risk factors and diagnosis }}$}

Peripheral arterial disease is most commonly caused by atherosclerosis, but it may also be a secondary disease to cardiac or vascular embolism, vasculitis, hypercoagulopathy, vascular dissection, vascular compression syndromes, and other disorders (2). The classical risk factors that are associated with more than $50 \%$ of PAD cases in the population include smoking, diabetes mellitus, dyslipidemia and hypertension, and prevalence increases with age (8). Diabetes is a particularly potent risk factor, as patients with diabetes have a risk of developing PAD that is four times the general population. Patients that smoke and have diabetes further increase that risk by 2.5 fold (5). After accounting for the classical risk factors, nearly $50 \%$ of PAD cases are caused by unmeasured factors that likely result from hundreds of genes interacting with each other and the environment (2). Several studies have been conducted in which inherited factors were concluded to contribute to the manifestation of PAD, and genetic variations have been discovered that are predictive of coronary artery disease and PAD (8-10). However, genetic variations that are more specific for PAD are unlikely to be found (2).

Despite having known risk factors, PAD is underdiagnosed since only $10 \%$ to $30 \%$ of PAD patients present with the classic symptom of intermittent claudication (2). Clinical studies have determined that asymptomatic patients are more likely to be diagnosed by noninvasive testing with the ankle-brachial index ( $\mathrm{ABI})$ test, but since many clinicians rely on claudication symptoms alone to detect PAD, $85 \%$ to $90 \%$ of PAD diagnoses may be missed (11). To measure the ABI, a Doppler ultrasound probe is used to measure the systolic blood pressures in the upper extremities at the brachial arteries and in the lower extremities at the 
dorsalis pedis and posterior tibial arteries $(8,11)$. The ABI is defined as the higher of the two ankle systolic pressures divided by the higher brachial systolic pressure. An ABI less than or equal to 0.9 is the criterion for diagnosis of $\operatorname{PAD}(8,11)$. Once $\mathrm{ABI}$ testing is adopted by more clinicians as a simple, non-invasive way to diagnosis PAD in asymptomatic patients with known risk factors, the next step will be to improve upon long term treatment of those affected by this disease.

\section{$\underline{\text { Prognosis and treatment }}$}

Even with its history of being underdiagnosed, PAD is a significant problem resulting in approximately half a million hospitalizations annually. Additionally, these patients receive less intensive treatment than patients with coronary artery disease due to lack of familiarity with PAD in the primary care setting $(2,11)$. Patients diagnosed with peripheral arterial disease face several symptoms that significantly affect quality of life in addition to the increased risk of heart attack and stroke, including intermittent claudication reducing mobility, morbidity due to non-healing ulcers and ischemic rest pain, and mortality through association with coronary and carotid artery disease (2).

Treatment options for PAD patients include management of risk factors, supervised exercise programs, pharmacotherapy, and surgical intervention $(5,12)$. Some risk factors, such as smoking, are relatively straightforward to address. Smoking is the dominant modifiable risk factor for PAD, and a dose-dependent relationship has been observed between smoking and severity of PAD (12). Poor wound healing, increased risk of amputation, and increased mortality are all linked to smoking, especially in diabetic patients. With smoking cessation the 10 year survival rate in diabetic PAD patients was $82 \%$, a 
significant improvement over the $46 \%$ survival rate of those who did not stop smoking (5). Exercise is another life-style change that can have an impact on symptoms for PAD patients. It significantly improves maximal walking time and overall walking ability in patients with stable intermittent claudication, and is more effective than both antiplatelet therapy and angioplasty (12). Other risk factors can be addressed with pharmacotherapy such as statin drugs, antihypertensive drugs and antiplatelet therapy which may reduce the incidence of claudication and increase maximum walking distance (12). Diabetes can be more complicated to address as a risk factor for PAD, since diabetic patients tend to have more diffuse vascular disease. Control of blood glucose levels has been shown to reduce microvascular complications of diabetic PAD, but there were no significant reductions in stroke, myocardial infarction or amputation due to PAD. Instead, blood pressure and blood cholesterol levels should be rigorously controlled with pharmacotherapy and life-style changes to prevent serious vascular events in diabetic PAD patients (12).

Surgical revascularization procedures are an option for patients that fail to improve with risk factor management and pharmacotherapy, have non-healing ulcerations, gangrene, or critical limb ischemia (5). These procedures include percutaneous transluminal angioplasty (PTA) and bypass surgery. PTA has been shown to restore adequate blood flow in patients with critical limb ischemia, and bypass grafts have been performed effectively on more distal arteries in the limb (6). Both types of revascularization have reduced rates of early major amputation in comparison with patients without revascularization (6).

Some patients, particularly those with diabetes or prior revascularization procedures, are poor candidates for surgical interventions. Diffuse atherosclerosis in multiple anatomical regions is common in diabetic PAD patients and difficult to treat, and repeat surgeries are 
technically prohibitive $(6,13)$. Thus, there is a significant, unmet need for alternative treatment strategies for patients with critical limb ischemia. Efforts to meet this need started as early as 1994 with a human clinical trial in which vascular endothelial growth factor (VEGF) was delivered as a naked plasmid intramuscularly by Baumgartner et al. This phase 1 clinical trial sought to test the concept of therapeutic angiogenesis in patients with critical limb ischemia after preclinical studies showed that angiogenic growth factors can stimulate collateral artery development. While the sample size of this study was small, they observed significant improvements in the $\mathrm{ABI}$, evidence of improved distal blood flow, and improvement in ischemic ulcers (14). Figure 1.1 illustrates the concept of therapeutic angiogenesis in which growth factors are delivered to promote remodeling and growth of collateral vessels to compensate for the reduced blood flow caused by PAD.



Figure 1.1: Concept of therapeutic angiogenesis using DNA of growth factors (13).

Since this study, many other angiogenic growth factors have been tested in clinical trials including various forms of VEGF, fibroblast growth factor (FGF), and hepatocyte growth 
factor (HGF) $(15,16)$. As shown in Table 1.1, some of the early clinical trials have produced mildly promising results, but quantitative analysis of the efficacy of new treatments is lacking due to the challenges of choosing patients and measuring a true signal of bioactivity among the background noise of variability between patients with chronic critical limb ischemia (17). This is particularly true for patients with diabetes, a population in which therapeutic angiogenesis has not been studied enough. There is also a concern about the safety of growth factor administration in diabetic PAD patients, because they have a greater risk of widespread vascular disease including retinopathy which might be exacerbated by proangiogenic growth factors $(6,17)$. In non-diabetic patients with PAD, some adverse effects have been observed with therapeutic angiogenesis treatment, including hypotension, vascular leakage, and transient tissue edema (17). 
Table 1.1: Gene therapy in peripheral arterial disease: bioactivity data (17).

\begin{tabular}{|c|c|c|c|c|c|c|c|}
\hline Trial Design & Condition & $\begin{array}{l}\text { Treatment(s) } \\
\text { Dosing }\end{array}$ & Route & $\begin{array}{c}\text { Patients } \\
\text { Verum/Placebo, } \\
\mathrm{n}\end{array}$ & $\begin{array}{l}\text { Follow- } \\
\text { Up }\end{array}$ & $\begin{array}{l}\text { Parameters of } \\
\text { Bioactivity }\end{array}$ & $\begin{array}{l}\text { Trends for } \\
\text { Bioactivity }\end{array}$ \\
\hline \multicolumn{8}{|l|}{ Phase I/II } \\
\hline $\begin{array}{l}\text { DELTA- } \\
1 \text { phase II } \\
\text { double-blind, } \\
\text { placebo- } \\
\text { controlled, } \\
\text { multicenter trial }\end{array}$ & PAD; claudication & $\begin{array}{c}\text { Del-1 } \\
\text { plasmid } \\
\text { (VLTS-589) }\end{array}$ & $\mathrm{IM}$ & $52 / 53$ & $\begin{array}{c}30-90- \\
180 \mathrm{~d}\end{array}$ & $\begin{array}{c}\Delta \text { Walking time } \\
(\mathrm{PE}) ; \Delta \mathrm{ABI} ; \Delta \mathrm{COT} ; \\
\Delta \mathrm{QOL}\end{array}$ & None \\
\hline $\begin{array}{l}\text { PREVENT I phase } \\
\text { I double-blind } \\
\text { trial }\end{array}$ & $\begin{array}{l}\text { PAD/revascularization, } \\
\text { CLI, vein bypass graft }\end{array}$ & $\begin{array}{l}\text { E2F decoy } \\
\text { (edifoligide) }\end{array}$ & $\begin{array}{c}\text { Ex } \\
\text { vivo }\end{array}$ & $16 / 8 / 17$ & $12 \mathrm{mo}$ & Graft occlusion & $\begin{array}{l}\downarrow \text { Graft occlusion, } \\
\text { critical stenosis, } \\
\text { revision }\end{array}$ \\
\hline trial Phase I & PAD, CLI & $\begin{array}{c}\text { FGF- } 1 \\
\text { plasmid } \\
(\mathrm{NV} 1 \mathrm{FGF}) ; 1 \times \\
500-16000 \\
\mu \mathrm{g}, 2 \times 500- \\
8000 \mu \mathrm{g}\end{array}$ & $\mathrm{IM}$ & $\begin{array}{l}\text { Safety } 51 / 0 \\
\text { efficacy } 15 / 0\end{array}$ & $3-6 \mathrm{mo}$ & $\begin{array}{c}\mathrm{TCPO}_{2}, \mathrm{ABI}, \mathrm{TBI}, \\
\text { pain, ulcer healing }\end{array}$ & $\begin{array}{l}\uparrow \mathrm{TCPO}_{2}(P=0.01), \\
\uparrow \mathrm{ABI}(P=0.01), \\
\text { tpain }(P=0.001), \\
\text { । ulcer size } \\
\quad(P=0.01)\end{array}$ \\
\hline $\begin{array}{l}\text { PM202 } \\
\text { phase II double- } \\
\text { blind, placebo- } \\
\text { controlled, } \\
\text { multicenter trial }\end{array}$ & PAD, CLI & $\begin{array}{c}\text { FGF-1 } \\
\text { plasmid } \\
\text { (NV1FGF), 2- } \\
16 \mathrm{mg}\end{array}$ & IM & 71 & $\mathrm{~N} / \mathrm{A}$ & $\begin{array}{l}\triangle \mathrm{TCPO}_{2}(\mathrm{PE}), \mathrm{ABI} \text {, } \\
\text { TBI, ulcer healing, } \\
\text { amputation, death }\end{array}$ & $\begin{array}{l}\text { I. Death, } \\
\text { amputation }\end{array}$ \\
\hline $\begin{array}{l}\text { TALISMAN } 201 \\
\text { phase II double- } \\
\text { blind, placebo- } \\
\text { controlled, } \\
\text { multicenter trial }\end{array}$ & PAD, CLI & $\begin{array}{c}\text { FGF- } 1 \\
\text { plasmid } \\
\text { (NV1FGF), } 4 \times \\
4 \mathrm{mg}\end{array}$ & $\mathrm{IM}$ & $56 / 56$ & 25 wk & $\begin{array}{c}\text { Complete ulcer } \\
\text { healing (PE), ABI, } \\
\text { amputation, death }\end{array}$ & $\begin{array}{c}\text { IRisk of all } \\
\text { amputations } \\
(P=0.015), \downarrow \text { risk of } \\
\text { major amputations } \\
(P=0.015), \downarrow \text { risk of } \\
\text { death }\end{array}$ \\
\hline $\begin{array}{l}\text { Phase I } \\
\text { dose-escalation, } \\
\text { double-blind, } \\
\text { placebo- } \\
\text { controlled trial }\end{array}$ & PAD, claudication & $\begin{array}{c}\text { rh bFGF, } 1 \times \\
10,1 \times 30 \\
2 \times 30 \\
\mathrm{mg} / \mathrm{kg}\end{array}$ & IA & $4-5-4 / 6$ & $6 \mathrm{mo}$ & $\begin{array}{c}\text { Calf flow } \\
\text { (plethysmography) }\end{array}$ & $\begin{array}{l}\text { ¿Blood flow } \\
(P=0.002)\end{array}$ \\
\hline $\begin{array}{l}\text { Phase II } \\
\text { dose-escalation, } \\
\text { double-blind, } \\
\text { placebo- } \\
\text { controlled trial }\end{array}$ & PAD, claudication & $\begin{array}{l}\text { rh bFGF, } 6 \times x \\
2 \mu \mathrm{g} \cdot \mathrm{kg}^{-1} \\
\cdot \mathrm{wk}^{-1}\end{array}$ & IV & $\begin{array}{l}16 / 8 \\
\text { (prematurely } \\
\text { terminated) }\end{array}$ & $\begin{array}{c}4-8-12 \\
w k\end{array}$ & PWT (PE), QoL & None \\
\hline $\begin{array}{l}\text { TRAFFIC } \\
\text { phase II, double- } \\
\text { blind, placebo- } \\
\text { controlled trial }\end{array}$ & PAD, claudication & $\begin{array}{c}\text { rh FGF- } 2,1 \times \\
\text { or } 2 \times 30 \\
\mu g / \mathrm{kg}\end{array}$ & IA & $66-61 / 68$ & $\begin{array}{c}90-180 \\
\mathrm{~d}\end{array}$ & $\begin{array}{l}\text { PWT (PE), } \triangle \mathrm{PWT} \text {, } \\
\text { COT, } \triangle \mathrm{ABI}, \mathrm{QOL}\end{array}$ & $\begin{array}{l}1 \text { Change in PWT } \\
\text { (day } 90, P=\mathrm{s} \text { ), } \uparrow \mathrm{ABI} \\
\text { (day } 90, P=\mathrm{s} \text { ) }\end{array}$ \\
\hline Phase & PAD/Buerger's, CLI & $\begin{array}{l}\text { bFGF/gelatin } \\
\text { hydrogel } \\
\text { sphere, } 200 \\
\mu \mathrm{g}\end{array}$ & $\mathrm{IM}$ & $7 / 0$ & $\begin{array}{c}4-24 \\
w k\end{array}$ & $\begin{array}{c}\text { 6-min Walk, } \\
\mathrm{TcPO}_{2} \text {, rest pain, } \\
\mathrm{ABI} \text {, ulcer healing, } \\
\text { perfusion (laser } \\
\text { Doppler), } \\
\text { thermography }\end{array}$ & $\begin{array}{c}\text { idistance for } 6- \\
\text { min walk } \\
(P=0.023), \uparrow \mathrm{TCPO}_{2} \\
(P=0.03), \downarrow \text { rest } \\
\text { pain }(P=0.022), \\
\text { improved ulcers } \\
(5 / 6), \uparrow \mathrm{ABI}(4 \mathrm{wk}, \\
P=0.024), \uparrow \mathrm{limb} \\
\text { perfusion } \\
(P=0.015), \uparrow \mathrm{limb} \\
\text { temperature }(4 \mathrm{wk}, \\
P<0.05)\end{array}$ \\
\hline $\begin{array}{l}\quad \text { Phase } \\
\text { I/II dose- } \\
\text { escalation, } \\
\text { double-blind, } \\
\text { placebo- } \\
\text { controlled, } \\
\text { multicenter trial }\end{array}$ & PAD, CLI & $\begin{array}{l}\text { Ad5FGF-4, } \\
2.87 \times 10^{8}- \\
10^{10} \mathrm{VP}\end{array}$ & $\mathrm{IM}$ & $10 / 3$ & $12 w k$ & $\begin{array}{l}\text { Rest pain, } A B I, \\
\text { angiography } \\
\text { (DSA), perfusion } \\
\text { (MRI, scintigraphy) }\end{array}$ & $\begin{array}{l}\text { \Rest pain }(4 / 10), \\
\text { more } / \text { bigger } \\
\text { vessels }\end{array}$ \\
\hline
\end{tabular}


Despite the lack of a clinical breakthrough in therapeutic angiogenesis treatment to date, there are several reasons why researchers continue to pursue this problem so that quality of life can be improved for patients who do not respond to risk factor management or qualify for surgical intervention. Work in this field is motivated by the fact that single genes appear to activate potent angiogenic mechanisms, and these effects may be magnified by activating specific targets and pathways with therapeutic agents. Additionally, novel delivery methods can be designed to direct therapeutic agents to the disease locus and potentially reduce the side effects observed in previous studies. These novel delivery strategies, along with a search for the most efficient and safe combination of proangiogenic genes and/or proteins, are currently being pursued in preclinical studies (17).

\section{Preclinical Models and Methods}

$\underline{\text { Introduction }}$

The mouse hind limb ischemia (HLI) model (18) has been used to study postnatal blood vessel formation and plays a vital role in testing new therapeutic angiogenesis approaches before they can be advanced to clinical trials. Typically, the femoral artery and vein are isolated from the femoral nerve after making a small incision in the hind limb. The artery and vein are then ligated with sutures at one or two locations. Depending on the severity of ischemia desired for the experiment, a segment of the vessels between the ligation points may be excised. These studies generally use a combination of several techniques to obtain information about the morphological and functional responses of the vasculature after induction of ischemia. In addition to studying the effect of therapeutic 
angiogenesis treatments on wild type mice, mouse models of diabetes and genetically modified strains can be included in these studies to better understand the mechanisms of vascular recovery in the context of conditions believed to impair angiogenesis and increase the severity of PAD (19-21).

\section{Current techniques for evaluating vascular recovery}

Despite the widespread use of the mouse hind limb ischemia model, current techniques for evaluating vascular recovery suffer from several limitations. Microcomputed tomography (micro-CT) permits global analysis of the vascular network in the entire limb and provides highly quantitative 3D data; however, it requires postmortem perfusion with contrast agents making it inefficient for longitudinal studies (2224). Similarly, x-ray angiography provides visualization of collateral arteries but it too is a post-mortem technique (25). Histology can be used to study microvessel density, but it is post-mortem, 2D and subject to sampling error. Laser Doppler perfusion imaging (LDPI) provides a non-invasive, functional measure of blood flow, but it is superficial and provides a relative rather than absolute measurement (18). A similar relative measure of recovery can be acquired with optical spectroscopy, which provides a point-based measurement of the oxygen saturation of hemoglobin based on the differing absorption spectra of oxyhemoglobin and deoxyhemoglobin at specific wavelengths $(19,25)$. Other methods for measuring tissue oxygenation at the microvascular level are more quantitative and include polarographic electrodes and fluorescence lifetime needle-based sensors, but these devices have several limitations including small sampling area, long stabilization periods, and invasiveness (26). Measurements of $\mathrm{pO}_{2}$ can also be obtained 
less invasively with the electron paramagnetic resonance (EPR) oximetry method, but this requires injection of an exogenous material (27). While each of these techniques provides important endpoints for hind limb ischemia studies, access to multiple instruments and multiple cohorts of mice are required to study the effect of novel therapeutic treatments over time. A complete picture of the structure-function relationships involved in the vascular response to ischemia is not currently attainable since robust quantitative endpoints cannot be obtained intravitally from a given mouse over time.

$\underline{\text { Motivation }}$

The limitations of current methodologies used to study the mouse hind limb ischemia model highlight a significant need for an improved tool for researchers in this field. Experimentation with post-mortem methods is expensive, time consuming, and subject to inter-animal variability that exists even within the same strain (28). An intravital, noninvasive imaging technique would be advantageous for developing novel therapeutic treatments for PAD, as it would accelerate throughput and decrease costs of basic studies. In addition to being non-invasive, this new technique should provide quantitative, absolute measures of recovery which may lead to new insight into the physiological processes of arteriogenesis and angiogenesis. Ultimately, this intravital data could lead to the development of more effective treatments for PAD by enabling a more robust analysis of the response to regenerative therapies. 


\section{Hyperspectral Imaging}

Non-invasive optical imaging methods have the potential to provide longitudinal data on vascular structure, oxygenation and hemodynamics. Hyperspectral imaging has been used to quantify hemoglobin oxygen saturation in vivo by collecting absorption images at multiple wavelengths and fitting the resulting absorption curve at each pixel using a modified version of Beer's Law $(29,30)$. This technique is quantitative and can provide 2-D spatial maps of blood oxygenation in terms of hemoglobin oxygen saturation with relative technical ease compared to polarographic electrodes and other needle-based $\mathrm{pO}_{2}$ sensors (29).

Hyperspectral imaging has been successfully applied to studies on tumors, brain imaging, and diabetic foot ulcers $(29,31-33)$. Previous studies have used a liquid crystal tunable filter (LCTF)-based system for collection of the specific wavelengths in a hyperspectral data set $(29,33,34)$. The collection leg of an LCTF-based hyperspectral system generally consists of the filter, a collection lens, and a CCD camera. The hyperspectral data is constructed by acquiring a CCD frame for each wavelength of interest as the filter tuning wavelength is swept across the range of interest. To determine hemoglobin saturation from hyperspectral images, absorption images are collected at multiple wavelengths, typically from $500 \mathrm{~nm}$ to $620 \mathrm{~nm}$ in 5 or 10 -nm increments, and used to generate an absorption curve for each pixel in the image. The excitation source for this wavelength range can be a simple halogen lamp providing white light that includes the wavelengths of interest. The wavelength range of $500-620 \mathrm{~nm}$ is used to measure 
hemoglobin absorption because this wavelength range contains important features unique to the absorption spectra of oxyhemoglobin $\left(\mathrm{HbO}_{2}\right)$ and deoxyhemoglobin (Hb-R) (Fig. 1.2).

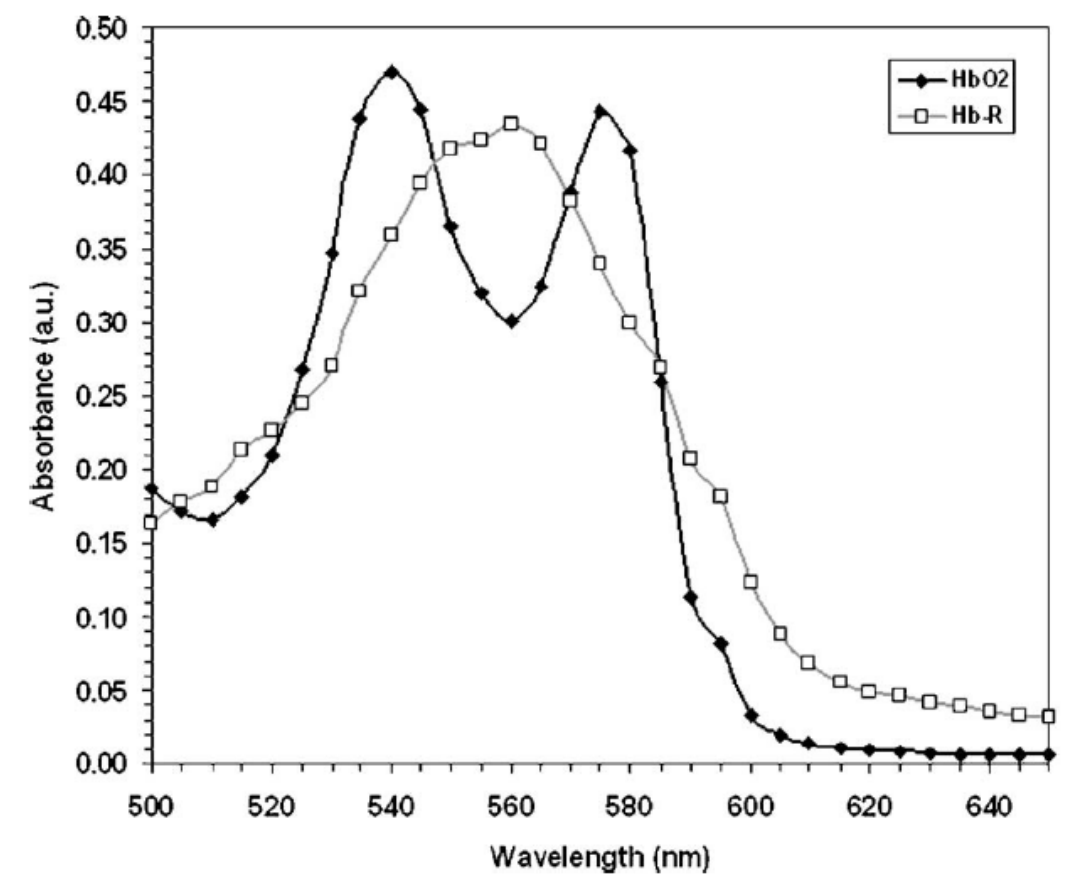

Figure 1.2: Absorbance spectra of oxy- and deoxyhemoglobin (29).

After the absorption spectrum for each pixel has been generated from the hyperspectral images, the spectrum is fit to a model equation (Eq. 1.1) derived from a modified version of Beer's Law (31):

$$
A_{\lambda}=\log \left(\frac{I_{0}}{I}\right)=\varepsilon_{\lambda}^{H b O_{2}} \cdot\left[H b O_{2}\right] \cdot L+\varepsilon_{\lambda}^{H b R} \cdot[H b R] \cdot L+S L
$$

where $A_{\lambda}$ is the absorbance at wavelength $\lambda, I$ is the pixel value, $I_{0}$ is the pixel value of reference light, $\varepsilon_{\lambda}{ }^{\mathrm{HbO2}}$ and $\varepsilon_{\lambda}^{\mathrm{HbR}}$ are the extinction coefficients for $\mathrm{HbO}_{2}$ and $\mathrm{HbR}$ and 
wavelength $\lambda$, respectively, $\left[\mathrm{HbO}_{2}\right]$ and $[\mathrm{HbR}]$ are the concentrations of oxy- and deoxyhemoglobin, respectively, $L$ is the path length, and $S$ is a path length-dependent scattering term (29). Tissue absorption in the absence of blood is computed as an estimate of $I_{0}(35)$. In this model, oxy- and deoxyhemoglobin are assumed to be the dominant absorbers in blood which is reasonable in the wavelength range selected. The model equation is solved using linear least squares regression to determine the relative concentrations of oxyhemoglobin and deoxyhemoglobin in each pixel, and hemoglobin saturation is computed as defined by Equation 1.2 after the path length terms cancel out:

$$
\mathrm{HbSat}=\frac{\left[\mathrm{HbO}_{2}\right]}{\left[\mathrm{HbO}_{2}+\mathrm{HbR}\right]} \cdot 100 \%
$$

The end result is a two dimensional map of hemoglobin oxygen saturation which can be visualized with a pseudocolor scale.

\section{Optical Coherence Tomography}

Optical coherence tomography (OCT) is a high resolution, three dimensional optical imaging technique that can resolve structures in tissue at depths around $2 \mathrm{~mm}$ (36-38). Cross sectional images are acquired in OCT by resolving the difference in path lengths between reflecting and scattering sites in the sample and that of a reference arm using a Michelson interferometer. OCT is used in a wide variety of applications, including imaging the retinal vasculature (39) and characterizing plaques in the coronary artery (40). Doppler OCT, a functional extension of OCT, is capable of imaging microvessel networks and blood flow 
velocities in three dimensions. Doppler shifts arise from motion in the sample (such as flowing red blood cells), and Doppler OCT detects this motion through phase-sensitive detection of interference between the probe and reference beam (37). Quantification of flow velocity and a vascular index from Doppler OCT data is described in Chapter 2.

In order to apply OCT techniques to a study of the mouse hind limb ischemia model, several challenges must be addressed. One significant challenge is imaging through the skin and obtaining repeatable measurements throughout a time course. Until recently, OCT had not been utilized to image skeletal muscle vasculature. However, when Jia et al. demonstrated the feasibility of using OCT to study the vasculature in the mouse hind limb they exposed the muscle with a skin incision during imaging (41). Ideally, this invasive step would be avoided in time course experiments so that repeat incisions would not be made and a wound response would not be evoked upon healing of the incision. In Chapter 2, images of the hind limb vasculature were obtained using OCT without a skin incision and fiducial markers were used to register images between time points over a three week time course.

Another challenge associated with imaging the hind limb vasculature is bulk motion artifact. This artifact is due to the animal's breathing and heartbeat, and it can significantly affect the ability to resolve vessels in a Doppler phase shift image. Several groups have established image processing techniques to address this problem and significantly reduce the bulk motion artifact in Doppler images. One such method is a histogram-based algorithm in which a histogram of the phase shift in all of the pixels of an A-scan is plotted. It is assumed that the blood vessels are small relative to the entire scan depth, and the bin with the largest pixel count will be from stationary tissue that may have a bulk shift due to motion artifact. 
The phase shift value for the bin with the largest pixel count is then subtracted from the rest of the image so that the phase shift for the stationary tissue is $0(42,43)$. This method is employed in the Doppler OCT image processing in Chapter 2.

In order to acquire both functional and morphological endpoints using OCT, multiple image acquisition and processing techniques can be used. Speckle variance OCT is advantageous for visualizing the morphology of blood vessels in volume scans collected with OCT since it is independent of the angle of blood flow relative to the imaging beam (unlike Doppler OCT). To generate speckle variance images, repeated images of the same location are acquired and the variance between repeated scans is computed. Fluid motion, i.e. blood flow, causes the speckle variance to be greater within blood vessels than in the surrounding stationary tissue, making it straightforward to visualize the vascular morphology in three dimensions or as a two dimensional projection of all of the vessels contained within a volume. Functional endpoints can be acquired using Doppler OCT, although Doppler OCT is sensitive to the angle between the direction of blood flow and the imaging beam. Therefore, several parameters must be optimized depending on the speed of blood flow in the vessels of interest. These parameters include the Doppler number (the number of phase shift differences averaged to produce a Doppler image), the angle of the hind limb, and the integration time for each A-scan. After optimization of image acquisition parameters, several quantitative endpoints can be extracted from a Doppler OCT image. These endpoints include the velocity profile across the center of a vessel of interest, the blood flow rate (a function of the mean velocity and the vessel diameter), the shear rate at the vessel wall, and a vascular index in which the fraction of pixels with a Doppler signal greater than a designated threshold is computed. Validation of the Doppler OCT 
measurement of fluid flow rate and some of these in vivo endpoints are discussed in Chapter 2.

In the work presented in Chapter 2, Doppler OCT and hyperspectral imaging techniques have been applied to a study of the vascular response in the mouse hind limb ischemia model. The results in Chapter 2 demonstrate the ability to acquire quantitative, intravital endpoints using these optical imaging modalities, validating further development of these methods as a new tool for evaluating novel therapeutic treatment strategies for peripheral arterial disease. 


\section{CHAPTER 2}

\section{QUANTITATIVE, INTRAVITAL OPTICAL IMAGING OF VASCULAR STRUCTURE AND FUNCTION IN A MODEL OF PERIPHERAL ARTERIAL DISEASE}

\section{Introduction}

An estimated 8 million American adults are affected by peripheral arterial disease (PAD) which is associated with significant morbidity and mortality (1). PAD is an occlusive arterial disease of the lower extremities and is associated with increased risk of myocardial infarction and stroke and reduced quality of life $(3,4)$. Therapy for PAD patients consists of modification of risk factors, exercise, pharmacotherapy, and surgical revascularization (5). However, some patients may have prior revascularization procedures or diffuse atherosclerosis and therefore be poor candidates for surgical intervention (6).

Development of collateral vessels can functionally compensate for obstructed arteries in patients with PAD, providing motivation for vascular biologists to intensely study the mechanisms that control formation and remodeling of vasculature. Complementary research on "therapeutic angiogenesis" seeks to develop clinical approaches for manipulating these mechanisms to stimulate formation of new blood vessels that will "naturally" bypass diseased arteries and abrogate the symptomatic effects of PAD (44). Animal models of peripheral limb ischemia play a vital role in the preclinical research efforts to test the efficacy of growth factor, gene, or cell based 
therapeutic angiogenesis approaches. In particular, the mouse hind limb ischemia (HLI) model (18) is used extensively both in basic science efforts to define the mechanisms involved in postnatal blood vessel formation and in testing of new therapeutic strategies.

While the utility of the hind limb ischemia model is well-established, no single available methodology currently used to assess vascular growth in this model allows acquisition of multi-functional, quantitative information from the living mouse. Current techniques used to characterize vessel remodeling and restoration of perfusion include histology, laser Doppler perfusion imaging (LDPI) and microcomputed tomography (micro-CT). Histology enables high resolution quantification of collaterals and capillaries, but this method is post-mortem, 2-D, and subject to sampling error. LDPI provides a non-invasive measure of functional recovery in terms of blood perfusion, but it is semi-quantitative and superficial. The micro-CT strategy produces high resolution, quantitative morphological data in 3-D, but it requires contrast agents and post-mortem analysis. Multiple cohorts of mice and access to multiple instruments are required to complete time-course analyses and obtain a complete picture of the vascular response using these methods, making experimentation both expensive and time-consuming. This fact highlights the significant, unmet need for an improved methodological toolbox for angiogenesis researchers. New techniques should enable quantification of absolute parameters of recovery and be non-invasive, thus allowing for individual animals to be longitudinally tracked for more robust analysis of the response to regenerative therapies.

Hyperspectral imaging is an optical imaging technique that has been successfully applied to the study of hemodynamics in several models including tumor angiogenesis (29), brain imaging (31), and diabetes mellitus (32). This technique is a method of 
scanning spectroscopy that quantifies hemoglobin oxygen saturation in vivo by collecting absorption images at multiple wavelengths $(500-620 \mathrm{~nm}$ in $10-\mathrm{nm}$ increments) and fitting the resulting absorption curve at each pixel using a modified version of Beer's Law (29). Point-based optical spectroscopy methods have long been employed to probe hemoglobin oxygen saturation in vivo, although these methods do not provide spatial registration or absolute quantification of data (25). Optical coherence tomography (OCT) is another optical method that is ideal for obtaining structural and functional endpoints for changes in microvasculature. OCT is a three dimensional optical imaging technique that can non-destructively visualize structural features in tissue at cellular-level resolution, with imaging depths $(\sim 2-3 \mathrm{~mm})$ that exceed current limits of microscopy. The speed of OCT has increased greatly in recent years, which allows for image volumes to be collected quickly ( $2 \times 2 \times 2 \mathrm{~mm}$ volume in 12 seconds (45)). Doppler OCT is a functional extension of OCT that images microvessel networks and blood flow velocities in 3-D $(37,38)$. Doppler shifts arise from motion in the sample (such as flowing red blood cells), and Doppler OCT detects this motion through phase-sensitive detection of interference between the sample and reference arms. The phase in the interferogram is especially sensitive to changes in optical path length, with typical sensitivities of $\sim 45$ picometers $(33,46,47)$. OCT has been used a wide variety of applications including human retinal circulation (48), dermatology (49), and tumor models (33), but it has more recently been applied to studies in skeletal muscles (41).

In the present study, our objectives were to use a liquid crystal tunable filter-based hyperspectral imaging system to obtain two dimensional images of hemoglobin oxygen saturation and study hypoxic recovery in the mouse hind limb ischemia model over time. 
Here, a distal site (the paw) was used in order to measure a region with the most pronounced hypoxia. This technique was compared with two current "gold standard" techniques: LDPI and tissue oxygen tension. Additionally, spectral domain Doppler OCT techniques were applied to obtain both functional and morphological data intravitally at the resolution of individual vessels in the hind limb. The combined application of these non-invasive optical imaging modalities provides researchers using the HLI model to test novel treatments for PAD with a new quantitative toolset for evaluating important, functional measures of arteriogenesis and hypoxic recovery intravitally.

\section{Materials and Methods}

$\underline{\text { Animals }}$

Male A/J mice were purchased from Jackson Laboratories (Bar Harbor, ME). This strain was selected because of its ischemic limb collateralization phenotype (50). All mice were between 8 and 10 weeks of age. The animals were fed a standard chow diet ad libitum and had free access to water. All protocols were approved by the Institutional Animal Care and Use Committee of Vanderbilt University and done in accordance with the National Institutes of Health Guide for the Care and Use of Laboratory Animals.

\section{$\underline{\text { Hind limb ischemia model }}$}

Hind limb ischemia was surgically induced in $n=12$ mice according to the wellestablished model of femoral artery ligation and transection $(18,50)$. Surgery was carried 
out under $1.5-2.5 \%$ isoflurane anesthesia with the mice maintained at normal body temperature. After administration of pre-operative analgesia (ketoprofen, $10 \mathrm{mg} / \mathrm{kg}$ ) and removal of hair, the surgical site was thoroughly cleansed with alternating iodine and chlorhexidine scrubs. A 5-mm unilateral incision was then made over the right medial thigh of the mouse. The femoral artery and vein were dissected away from the femoral nerve and were ligated with 6-0 silk sutures at two locations: immediately distal to the origins of the superficial epigastric artery and deep branch of the femoral artery, and proximal to the origin of a caudally branching vessel approximately $2 \mathrm{~mm}$ distal to the first ligation. The artery and vein were transected between the two ligations leaving a gap of 1-2 $\mathrm{mm}$. The wound was irrigated with sterile saline and the incision was closed with interrupted 5-0 nylon sutures. The contralateral limb served as the control for each animal. Analgesia (ketoprofen, $5-10 \mathrm{mg} / \mathrm{kg}$ ) was administered every $18-24$ hours postoperatively for 72 hours or until animals exhibited normal appearance and behavior.

\section{Hyperspectral imaging}

Two dimensional diffuse reflectance images were collected with a $200 \mathrm{~W}$ halogen lamp coupled into a $10-\mathrm{mm}$ core diameter liquid light guide for epi-illumination (schematic in Fig. 2.1). The light guide was mounted onto a Wild Heerbrugg M690 operating microscope (Leica). The collection head, also mounted on the operating microscope, consisted of a Varispec VIS-20 liquid-crystal tunable filter (LCTF) for bandlimited optical filtering (CRI, Inc.), a variable focal length ( $\mathrm{f}=28-80 \mathrm{~mm}$ ) camera lens (Nikon), and a 512 x 512 pixel PhotonMax CCD camera (Princeton Instruments) cooled to $-70^{\circ} \mathrm{C}$, resulting in a sampling density of $58 \mu \mathrm{m}$. The LCTF bandwidth is $10 \mathrm{~nm}$ 
when tuned to $550 \mathrm{~nm}$, but is narrower and wider at shorter and longer wavelengths, respectively. The transmission of the LCTF also increases at longer wavelengths. The LCTF tuning, CCD camera communication, shutter control and data handling were controlled with a custom computer program that enabled automated image acquisition with specifications of camera exposure time and gain. Images were saved as raw text files and converted to 16-bit grayscale uncompressed TIF files using Matlab software (The Mathworks, Incorporated).

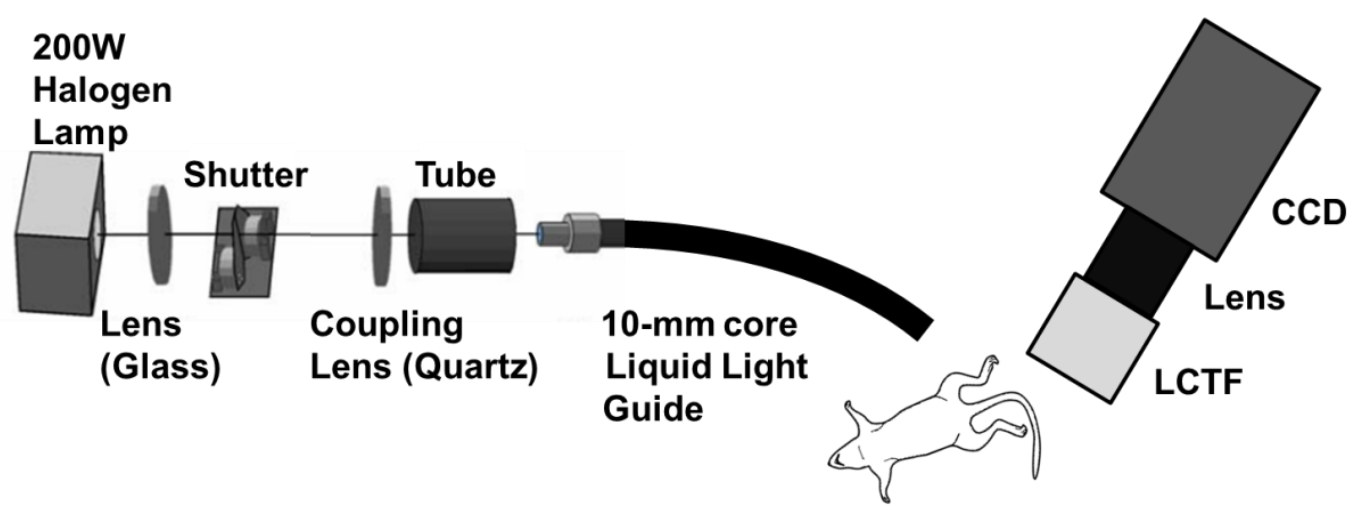

Figure 2.1: Schematic of hyperspectral system for diffuse reflectance imaging. White light from the halogen lamp is coupled into a liquid light guide for illumination of the footpads. Specific wavelengths are collected with a liquid crystal tunable filter (LCTF) mounted on the end of a variable focal length $(\mathrm{f}=28-80 \mathrm{~mm}$ ) camera lens and a $512 \times 512$ pixel CCD camera.

For validation of hemoglobin saturation mapping with this system, the spectra for pure oxyhemoglobin and deoxyhemoglobin solutions were measured with images acquired from 500 to $620 \mathrm{~nm}$ in 5 -nm intervals. Hemoglobin saturation images were calculated from calibrated hyperspectral data (corrected for dark offset and system response) using an extension of the Beer-Lambert law that solves for the hemoglobin saturation in each pixel using linear least-squares regression $(29,30)$. Oxygenated and deoxygenated hemoglobin were assumed to be the only factors contributing to absorption 
in this model. Quantification of hemoglobin oxygen saturation using the measured oxyand deoxyhemoglobin spectra to fit the data was validated with mouse whole blood in vitro at $100 \%$ and $0 \%$ oxygenation. Oxygenated blood samples were prepared by repeatedly inverting the sample in room air for several seconds. Deoxygenated blood samples were prepared with 5\% wt/vol sodium dithionite (29). Glass capillary tubes with an inner diameter of $1 \mathrm{~mm}$ were filled with the blood samples, sealed on the ends, and submerged in index-matching immersion oil on a glass slide. A diffuse reflectance standard was placed below the glass slide to provide total reflection in areas where light was not absorbed by hemoglobin. The saturation of oxygenated blood measured by the system was $99.9 \% \pm 0.3 \%$, and the saturation of deoxygenated blood was measured to be $6.7 \% \pm 3.5 \%$ (Fig. 2.2)

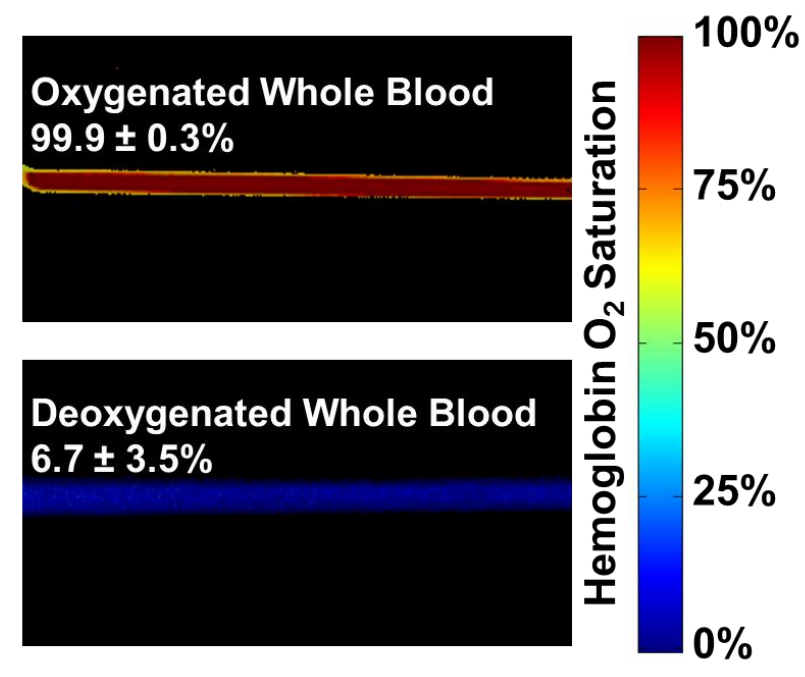

Figure 2.2: Validation of hemoglobin oxygen saturation measurement. Quantification of hemoglobin oxygen saturation was validated in vitro in glass capillary tubes with whole blood diluted with phosphate-buffered saline at $\mathrm{pH}$ 7.4.

Immediately following surgery (day 0 ) and at 7, 14 and 21 days post-surgery, control and ischemic footpads were imaged non-invasively through the skin with 
hyperspectral imaging. All imaging was carried out under 1-2\% isoflurane anesthesia with the mice maintained at normal body temperature. Anesthesia was delivered with $21 \%$ oxygen rather than $100 \%$ oxygen to avoid artificial elevation of normal hemoglobin saturation. Images of the control and ischemic footpads were acquired for wavelengths from 500 to $620 \mathrm{~nm}$ in 10-nm increments. Measurements of the dark offset and reflectance from a diffuse reflectance standard (Spectralon) were made before each imaging session for calibration. Hemoglobin saturation images of the footpads were calculated from hyperspectral data using Beer's Law as described for the validation experiment.

For quantitative analysis of hemoglobin saturation images, the regions of interest (ROI) in each image were defined by outlining the footpads using ImageJ software. Hemoglobin saturation values were quantified for each footpad by averaging the values for all pixels contained with the ROI. Hemoglobin saturation was analyzed as both an absolute value determined from the amounts of oxyhemoglobin and deoxyhemoglobin in each pixel and as a ratio of the ischemic footpad to the control footpad.

\section{Laser Doppler perfusion imaging}

Following hyperspectral imaging, perfusion in the footpads was measured by laser Doppler perfusion imaging (LDPI) (Perimed PSI) at 0, 7, 14 and 21 days postsurgery under isoflurane anesthesia and normal body temperature. The ambient light was consistent across all imaging days. At each time point, perfusion images were acquired with Perimed software at a rate of 4 frames per second, and 10 frames were averaged to provide perfusion values for the control and ischemic footpads. 
For analysis of perfusion data sets, the regions of interest (ROI) in each image were defined by outlining the footpads using Perimed software. Perfusion values were obtained for each footpad by averaging the values for all pixels contained with the ROI. Because LDPI is an intrinsically semi-quantitative and relative measurement, perfusion images were quantified as a ratio of the ischemic footpad to the control footpad.

\section{$\underline{\text { OxyLite } \mathrm{pO}_{2}} \underline{\text { measurements }}$}

Tissue oxygen tension $\left(\mathrm{pO}_{2}\right)$ was measured in the footpads of 5 mice at $0,7,14$ and 20 days post-surgery using the OxyLite (Oxford Optronix) tissue oxygenation sensor. To measure oxygen tension with this system, the probe tip was introduced into the footpad with a needle and pulses of LED light were transmitted along a fiber optic probe to excite a platinum-based fluorophore enclosed within a silicone matrix at the tip. The fluorescent light is quenched in the presence of oxygen and the instrument detects the fluorescence lifetime, which is inversely proportional to the concentration of dissolved oxygen. This value is used to calculate the absolute value of $\mathrm{pO}_{2}$ in $\mathrm{mmHg}$. The OxyLite system is most sensitive at low $\mathrm{pO}_{2}$ and simultaneously corrects for temperature effects with an integrated temperature probe providing the mouse core temperature during measurements. For this study, measurements were taken in two locations on each footpad. After insertion of the probe, the $\mathrm{pO}_{2}$ reading was allowed to stabilize for $10-15$ minutes. Once the reading stabilized, seven measurements were recorded over 30 seconds to obtain an average $\mathrm{pO}_{2}$ value for each location, and the two locations were averaged to provide a single value for each footpad. The $\mathrm{pO}_{2}$ data were analyzed as both absolute values in $\mathrm{mmHg}$ and as ratios of the ischemic footpad to the control footpad. 
Optical coherence tomography

A spectral domain OCT system with a central wavelength of $860 \mathrm{~nm}, 51 \mathrm{~nm}$ bandwidth, $50 \mu$ s minimum integration time per A line, lateral resolution of $25 \mu \mathrm{m}, 6.4$ $\mu \mathrm{m}$ axial resolution, and an imaging speed of about 20 frames/second with the selected imaging parameters was used in this study (Fig. 2.3). Prior to in vivo imaging of blood flow, measurement of flow rate with Doppler OCT was validated with an Intralipid phantom experiment for a range of flow rates controlled with a syringe pump. Briefly, $1 \%$ Intralipid was pumped through a 1-mm inner diameter glass capillary tube at flow rates ranging from 30 to $70 \mu \mathrm{l} / \mathrm{min}$. The OCT probe was aimed at the center of the tube and Doppler B-scans were collected for each flow rate. A three dimensional volume scan was collected to determine the angle of the tube relative to the imaging beam. The fluid velocity was computed from the measured Doppler phase shift using Equation 2.1:

$$
V=\Delta \emptyset \frac{\lambda_{o}}{4 \pi n \tau \cos (\alpha)}
$$

where $\Delta \varphi$ is the Doppler phase shift, $\lambda \mathrm{o}$ is the center wavelength, $\mathrm{n}$ is the refractive index, $\tau$ is the A-scan integration time, and $\alpha$ is the flow angle relative to the beam (measured from corresponding volume scan). Then, the flow rate was calculated using the peak of the second order polynomial curve fit to the velocity data and the cross sectional area of the capillary tube (Fig. 2.4). 


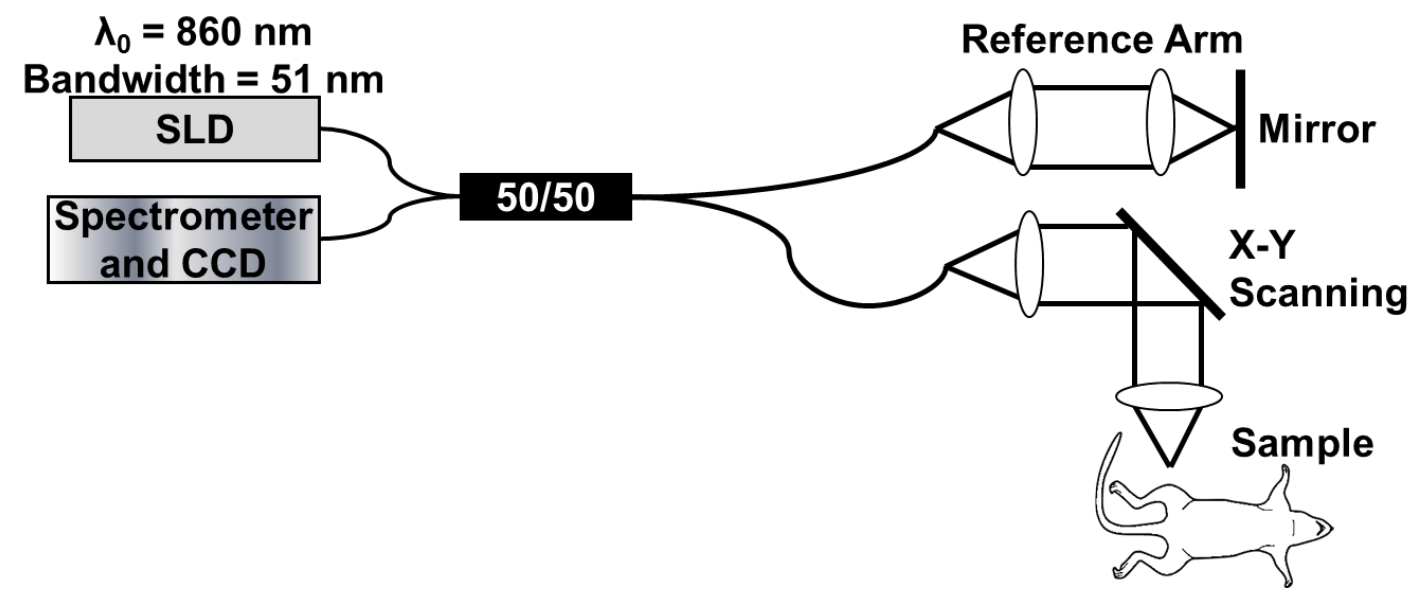

Figure 2.3: Schematic of spectral domain OCT system.
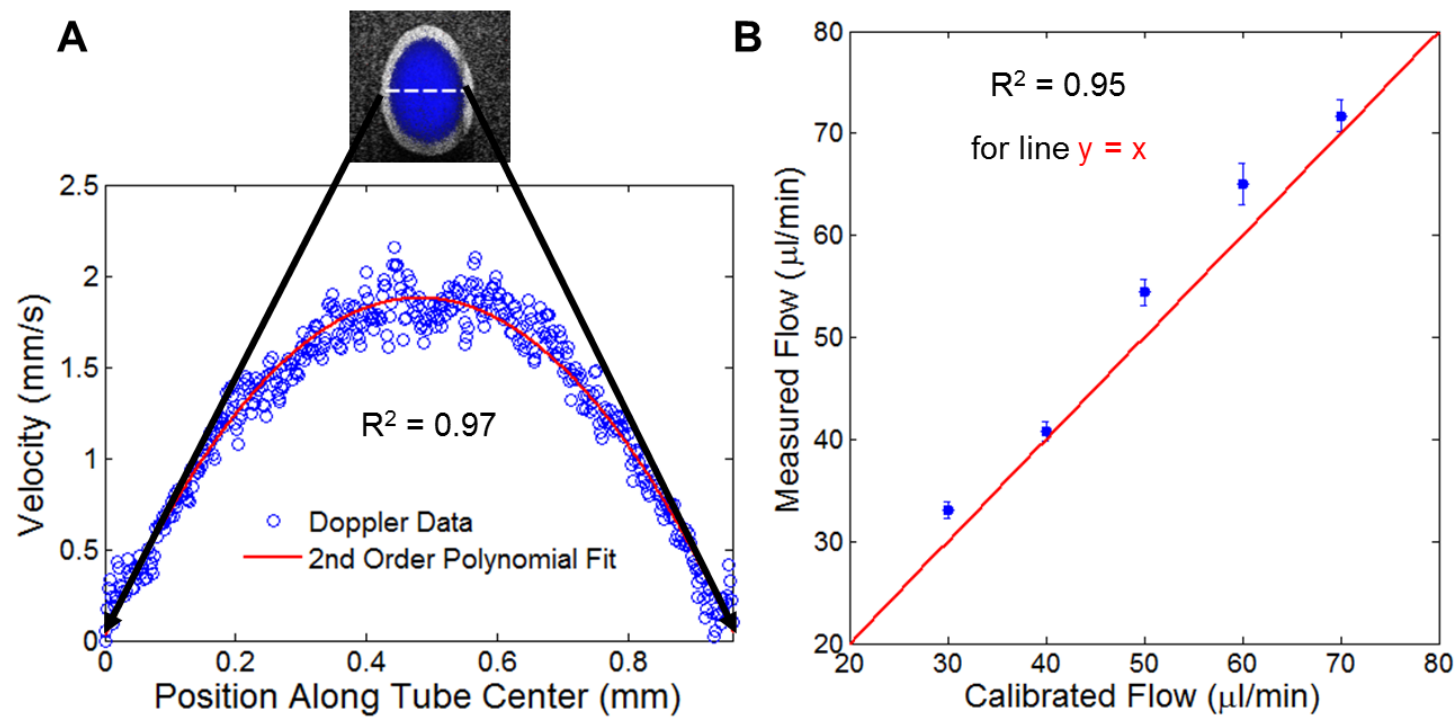

Figure 2.4: Doppler OCT validation. Measurement of flow rate with Doppler OCT was validated with an Intralipid phantom experiment for a range of flow rates. The measured flow rate was calculated using the peak of the curve fit and the cross sectional area of the capillary tube.

For the hind limb ischemia study, two dimensional B-scans and three dimensional volumes of Doppler OCT data were collected from three locations on each hind limb (ischemic and contralateral) after surgery for $n=4$ mice on days $0,7,14$ and 21 under 
isoflurane anesthesia and normal body temperature. The first area of interest crossed the femoral artery and vein distal to the ligation site and was selected to monitor restoration of blood flow to those vessels over time. The other two areas were located in the adductor muscle region medial to the incision site in order to monitor changes in the morphology and blood flow in collateral vessels. Repeated B-scans were collected in each area to allow for temporal averaging and reduction in noise. All OCT imaging was performed non-invasively through the skin of the hind limb.

For quantitative analysis of Doppler OCT B-scans containing the femoral artery and vein, the images were processed to reduce speckle noise and motion artifact. Briefly, Doppler noise due to signal fall-off in depth was excluded by an intensity threshold in the corresponding structural image, followed by minimization of the bulk motion artifact due to respiration with a histogram-based correction method (42). Repeated B-scans from the same location were filtered temporally, and then a Doppler threshold was computed from a region of static tissue (skin) to further separate blood flow detection from background noise. Pixels with a Doppler signal greater than the threshold were summed across the cross-sectional images and divided by the total pixel area to define a vascular index for the detected vasculature (43). Doppler volumes from the adductor muscle region were processed with the structural intensity threshold and bulk motion artifact correction steps, followed by a maximum signal projection in depth to visualize all collateral vessels present in the volume. 


\section{$\underline{\text { Statistical analysis }}$}

All data are presented as means \pm SEM. Statistical analyses were performed with Minitab software. Wilcoxon Signed-Rank statistics were computed for comparisons between ischemic and control limb measurements for each time point. The effect of time post-surgery on the response variables was tested with the Wilcoxon Signed-Rank test followed by a Bonferroni correction for multiple comparisons. Spearman's rank correlation coefficient was used to evaluate the hyperspectral imaging technique in relation to the gold standards of LDPI and OxyLite $\mathrm{pO}_{2} . \mathrm{P}<0.05$ was interpreted as significant in all analyses.

\section{Results}

Measures of vascular structure and function following induction of hind limb ischemia were obtained using optical imaging methods (hyperspectral imaging and optical coherence tomography) and compared to traditional methods of evaluating the mouse hind limb ischemia model (laser Doppler perfusion imaging and tissue oxygen tension).

\section{$\underline{\text { Perfusion measurements with LDPI }}$}

Perfusion in the footpads was analyzed in the same animals whose hemoglobin saturation was measured. The ischemic-to-control perfusion ratio was severely reduced at day 0 as shown by a representative perfusion image (Fig. 2.5A) then increased each week 
until perfusion was completely recovered in the ischemic limb (Fig. 2.5B). Significant increases in the ratio were observed between days 0 and 7 and between days 7 and 14 post-surgery $(\mathrm{p}<0.01)$. Between days 14 and 21 , perfusion was recovered in the ischemic footpad although the increase was not significant.
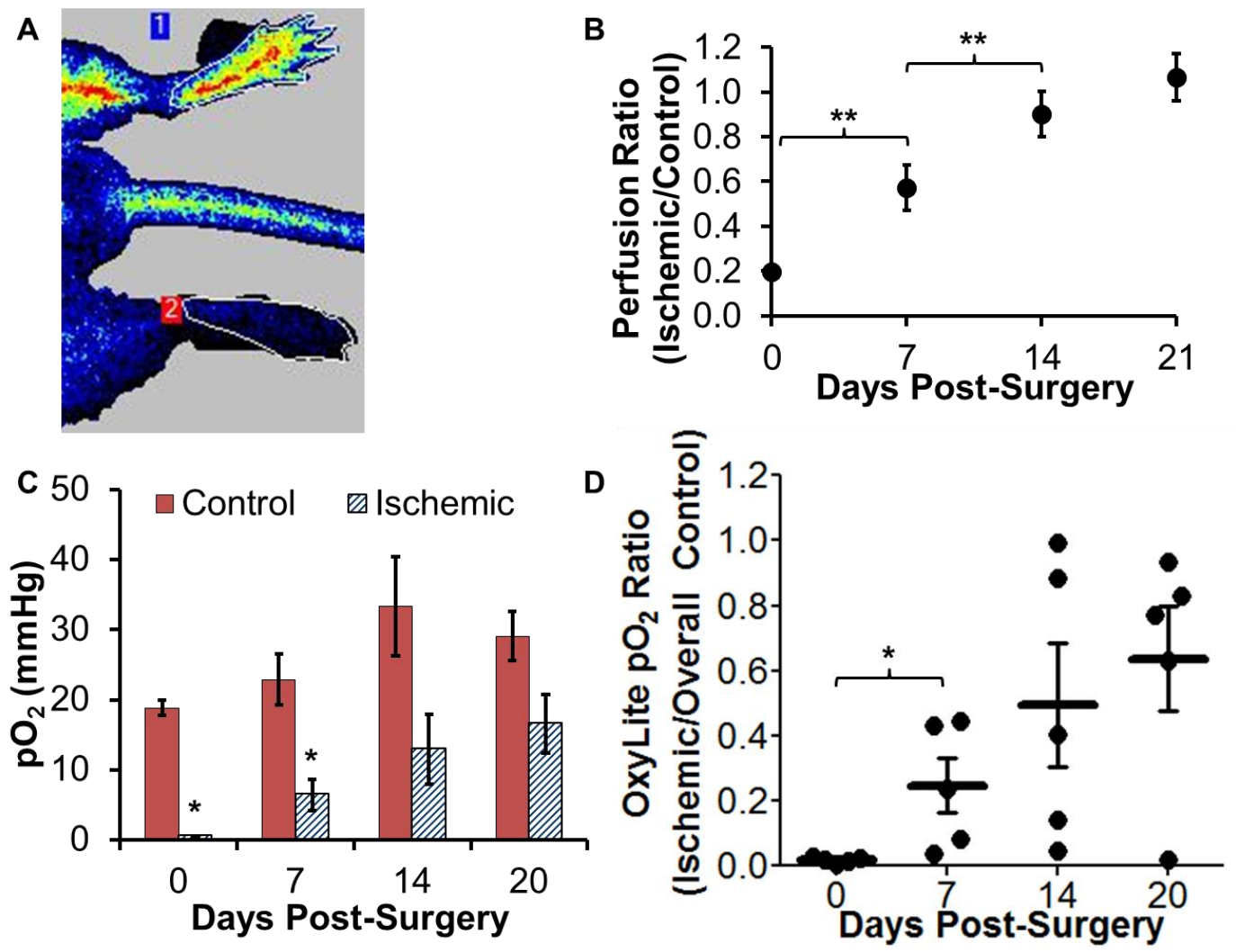

Figure 2.5: Measurements with LDPI and the OxyLite tissue oxygenation sensor confirm time course of recovery in ischemic limb. (A) Representative LDPI image of ischemic (bottom) and control (top) footpads post-surgery. (B) The normalized perfusion data demonstrate full recovery of perfusion in the ischemic footpad relative to the control footpad. (C) Absolute $\mathrm{pO}_{2}$ values from control and ischemic footpads measured with the OxyLite system (mean \pm SEM with $n=5$ ). (D) Individual animal ratios (ischemic $\mathrm{pO}_{2}$ normalized to overall control $\mathrm{pO}_{2}$ ) plotted with lines representing mean ratio $\pm \mathrm{SEM}$ at each time point. Data presented as mean \pm SEM with $n=7$. * corresponds to a one-sided $\mathrm{p}$-value $<0.05$ and $* *$ corresponds to $\mathrm{p}<0.01$ as determined by a Wilcoxon Signed-Rank test (B-D) followed by a Bonferroni correction for multiple comparisons (B, D). 


\section{Tissue oxygen tension in the hind feet}

The partial pressure of oxygen in the footpads as measured with the OxyLite $\mathrm{pO}_{2}$ sensor showed a significant difference between the control and ischemic footpads through day 7 (Fig. 2.5C). However, the ischemic-to-control footpad ratios were highly variable between animals (Fig. 2.5D). Figure 2.6 illustrates the high variation within control footpad measurements as a result of both location (two point measurements, $\mathrm{C} 1$ and C2) and the tendency of the OxyLite system to be less precise at "normal" $\mathrm{pO}_{2}$ levels (greater than $10 \mathrm{mmHg}$ ). These examples of location-specific $\mathrm{pO}_{2}$ data at days 0 (Fig. 2.6A) and 7 (Fig. 2.6B) also show that hypoxic $\mathrm{pO}_{2}$ values are more precisely measured by the sensor and that there is little temporal variation within the measurements taken over a 30 second period at each location. 


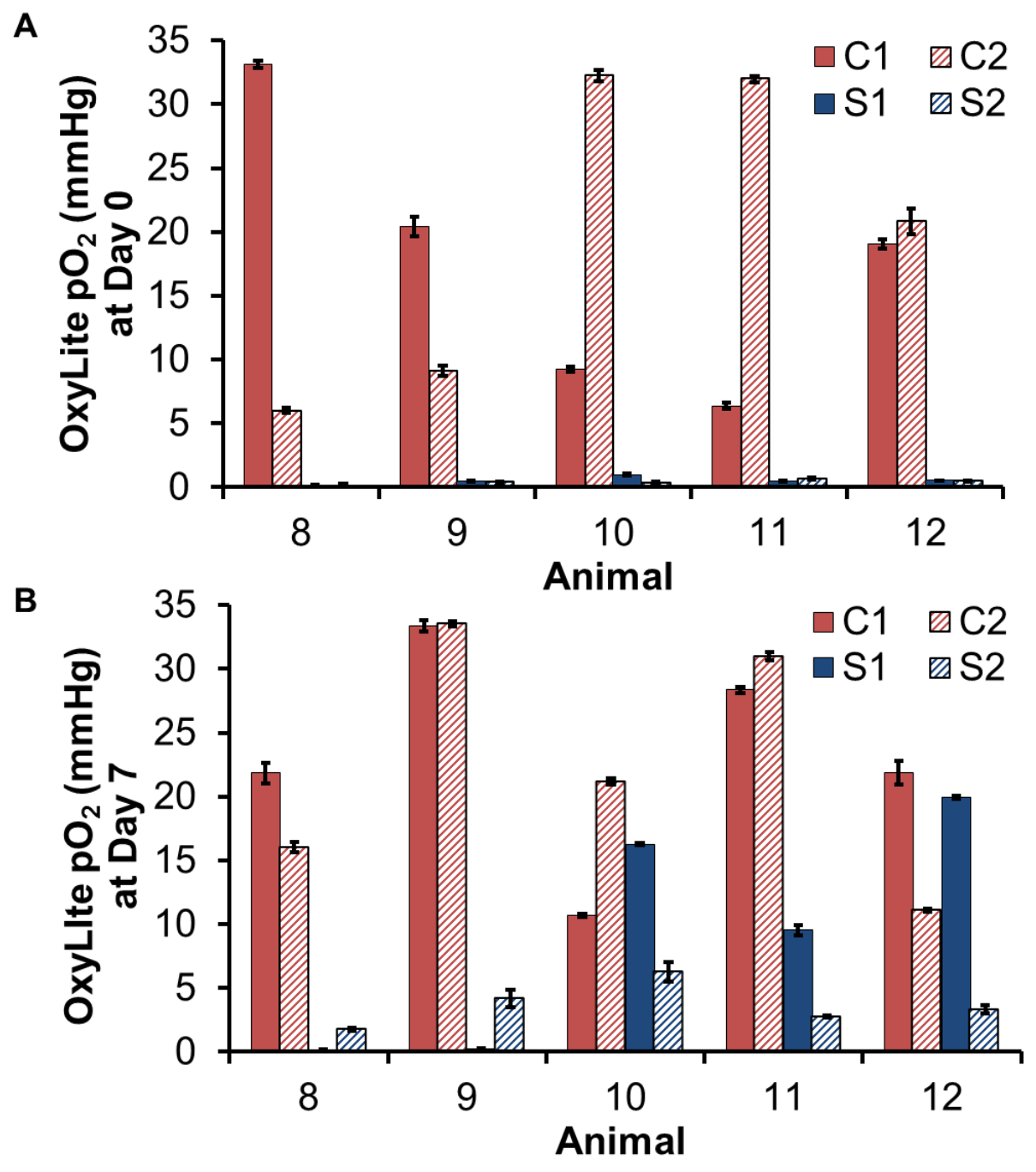

Figure 2.6: OxyLite tissue oxygenation sensor variability. Measurements are more precise at low tissue $\mathrm{pO}_{2}$ and show high spatial variability at normal $\mathrm{pO}_{2}$ levels. (A) $\mathrm{pO}_{2}$ data from each animal at day 0 for two locations on each footpad. (B) $\mathrm{pO}_{2}$ data from each animal at day 7. Data presented as mean \pm SDM of 7 readings in each location collected over 30 seconds. C1, C2: control footpad measurement 1 and 2; S1, S2: surgery footpad measurement 1 and 2 .

\section{Measurement of hemoglobin oxygen saturation}

We used an LCTF-based hyperspectral imaging system to non-invasively acquire quantitative hemoglobin saturation measurements from the hind feet of mice after induction of ischemia in the right hind limb. Hemoglobin saturation was measured for the 
same mice that were imaged with LDPI. This method provides a novel alternative to the invasive measurement of $\mathrm{pO}_{2}$ and the semi-quantitative nature of LDPI.

Analysis of hemoglobin saturation images of the hind feet revealed a significant reduction in oxygenation of the ischemic footpad compared to the control footpad from day 0 (representative image in Fig. 2.7A) through day 14 post-surgery $(\mathrm{p}<0.05)$ (Fig. 2.7B). Hemoglobin saturation in the ischemic footpad was reduced to approximately $40 \%$ of the control value immediately following surgery as shown by the normalized data (Fig. 2.7C), and the ratio increased significantly at each time point $(\mathrm{p}<0.01)$. The hemoglobin saturation ratio (ischemic to control footpad) increased over time to surpass $100 \%$ by day 21. In addition to quantification of the mean hemoglobin oxygen saturation value for each footpad, hyperspectral imaging provided an additional quantitative endpoint, the coefficient of variation across all of the pixels within the ROI, since it acquires a two dimensional image rather than a point-based measurement. The coefficient of variation was significantly greater in the ischemic footpad through day 14 of the time course $(\mathrm{p}<$ 0.05), showing that hemoglobin oxygenation is more uniform across the entire control footpad than the ischemic footpad. 

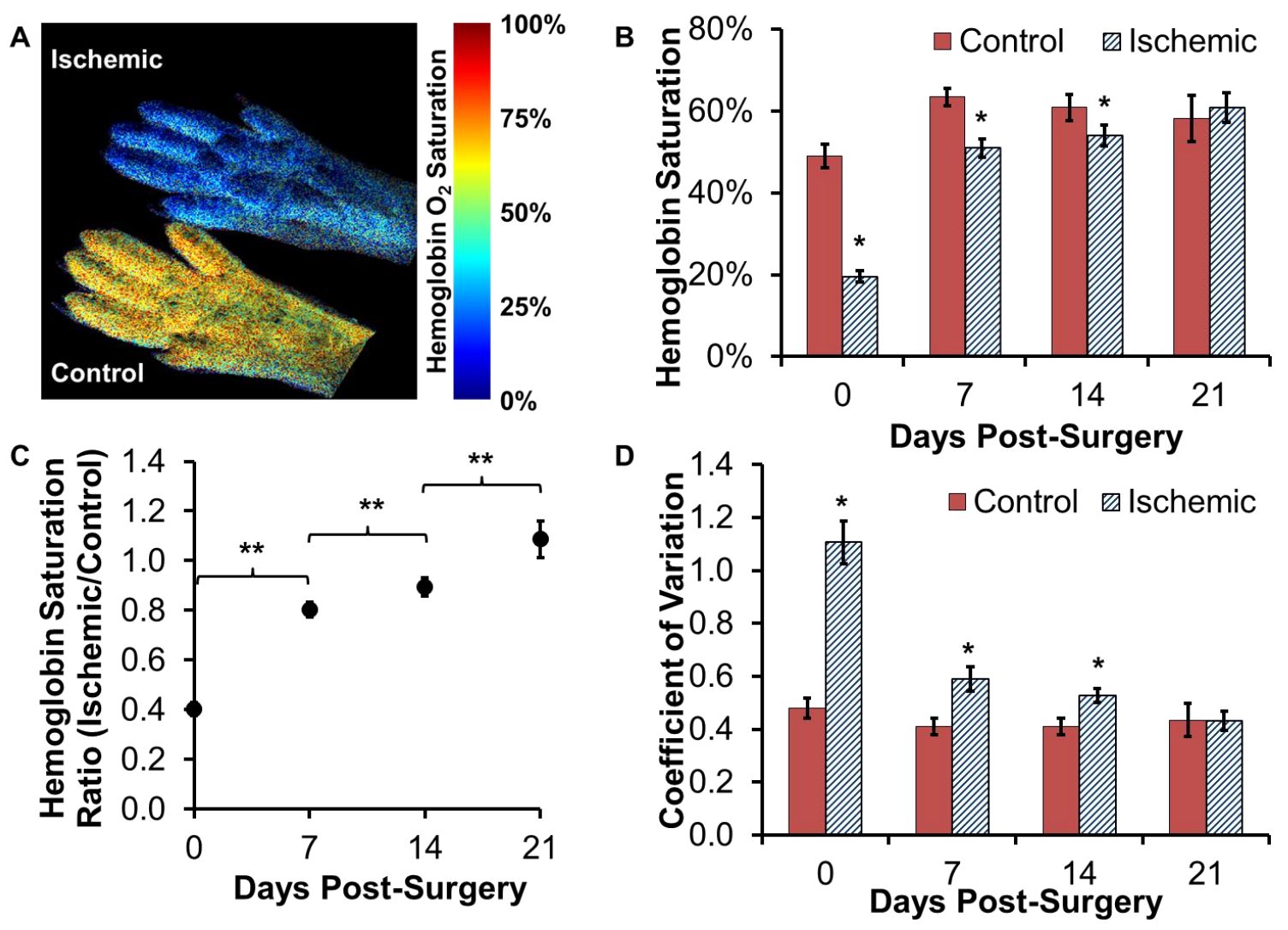

Figure 2.7: Hemoglobin oxygen saturation measured with hyperspectral imaging undergoes significant changes in the ischemic hind limb. (A) Representative hyperspectral image of the ischemic (top) and control (bottom) footpads after surgery. (B) Absolute hemoglobin saturation was quantified as the mean of all pixels within each footpad. There are significant differences between the ischemic and control footpads at days 0,7 and 14. (C) Data normalized to the control footpad demonstrate complete recovery from hypoxia in the ischemic footpad relative to the control footpad with significant increases in the ratio between successive time points. (D) The coefficient of variation is significantly greater in the ischemic footpad through day 14 of the time course. Data presented as mean \pm SEM with $\mathrm{n}=7$. * corresponds to $\mathrm{p}<0.05$ and ** corresponds to $\mathrm{p}<0.01$ as determined with a Wilcoxon Signed-Rank test (B-D) and a Bonferroni correction for multiple comparisons (C). P-values for (C) are one-sided as the ratio is expected to increase with time.

In order to understand the underlying cause of the changes in hemoglobin saturation, we studied the changes in the relative amounts of oxygenated and 
deoxygenated hemoglobin (Fig. 2.8). There was a significant decrease in oxyhemoglobin $(\mathrm{p}<0.05)$ in the ischemic footpad compared to the control immediately after surgery which recovered to control levels by day 7 (Fig. 2.8A). Additionally, the concentration of oxyhemoglobin in the ischemic footpad surpassed that in the control footpad at day 21. Deoxyhemoglobin levels showed the opposite trend, with significantly higher amounts in the ischemic footpad relative to the control values from day 0 to day 14 ( $\mathrm{p}<0.05)$ (Fig. $2.8 \mathrm{~B})$. By day 21, the amount of deoxyhemoglobin in the ischemic footpad is no longer significantly greater than that in the control. When the hemoglobin values in the ischemic footpad are normalized to the control values (Fig. 2.8C), significant increases in the oxyhemoglobin ratio occur between days 0 and 7 and days 14 and $21(\mathrm{p}<0.05)$. Changes in deoxyhemoglobin are more delayed, with a significant change in the ratio occurring from day 7 to day 21 post-surgery $(\mathrm{p}<0.05)$. Additionally, total hemoglobin is greater in the ischemic footpad at days 14 and 21 ( $\mathrm{p}<0.05$ ) (Fig. 2.8D), which agrees with previous results (19). Taken together, these data suggest that changes in both oxyhemoglobin and deoxyhemoglobin contribute to the observed changes in hemoglobin saturation in the ischemic hind limb. 

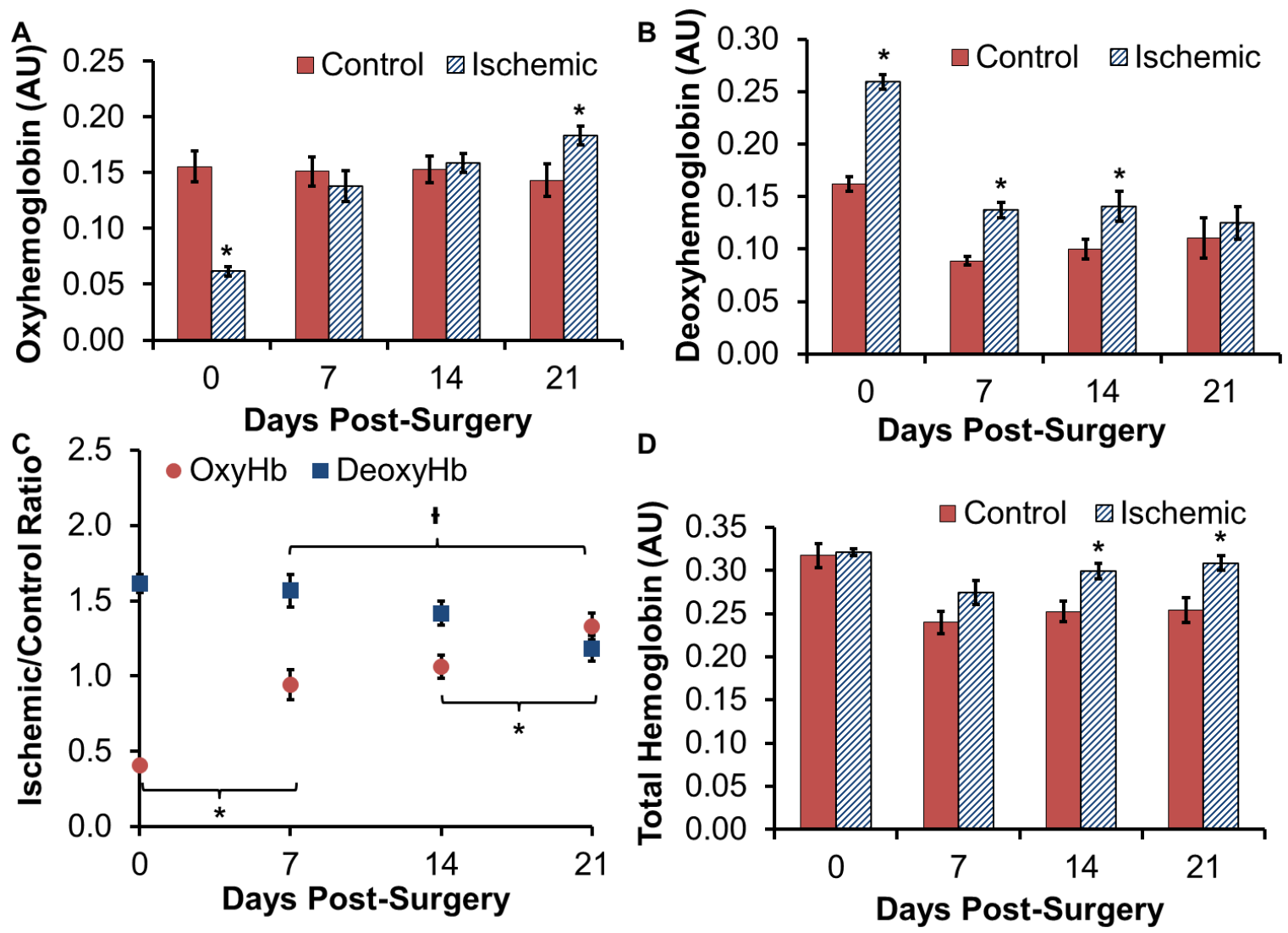

Figure 2.8: Trends in oxyhemoglobin and deoxyhemoglobin content after surgery. (A) The concentration of oxyhemoglobin is significantly decreased in the ischemic footpad in comparison to the control footpad at day 0 but surpasses the control at day 21 . (B) Deoxyhemoglobin is significantly higher in the ischemic footpad through day 14. (C) Relative oxyhemoglobin concentration in the ischemic footpad normalized to the control footpad shows significant increases in the first week and the last week of the time course. Deoxyhemoglobin concentration in the ischemic footpad normalized to the control shows a significant decrease from day 7 to day 21. (D) Total hemoglobin is significantly higher in the ischemic footpads at days 14 and 21. Values for each animal were obtained from the average of all pixels within each footpad. Data presented as mean \pm SEM with $n=7$. * and $¥$ correspond to $\mathrm{p}<0.05$ as determined with a Wilcoxon Signed-Rank test (A-D) and a Bonferroni correction for multiple comparisons (C). In (C), * refers to oxyhemoglobin data points and $\mathrm{x}$ refers to deoxyhemoglobin data.

Recovery of hemoglobin oxygen saturation in the footpads of the hind limbs followed a trend similar to that of the perfusion findings although the ischemic-to-control ratio measured by LDPI was reduced more severely at day 0 (Fig. 2.5A). The normalized 
data (ischemic/control) obtained with these two techniques correlated well with a Spearman's rank correlation coefficient of $0.81(\mathrm{p}<0.001)$ (Fig. 2.9A). The OxyLite $\mathrm{pO}_{2}$ measurements also indicated a trend toward recovery in the ischemic footpad, although the spatial variability and poor sensitivity at "normal" $\mathrm{pO}_{2}$ levels renders this measurement inconclusive in terms of showing complete recovery. Despite this variability, the mean ischemic-to-control ratios for hemoglobin oxygen saturation and tissue oxygenation follow a monotonically increasing function with a Spearman's rank correlation coefficient of 1 ( $\mathrm{p}<0.1)$ (Fig. 2.9B).
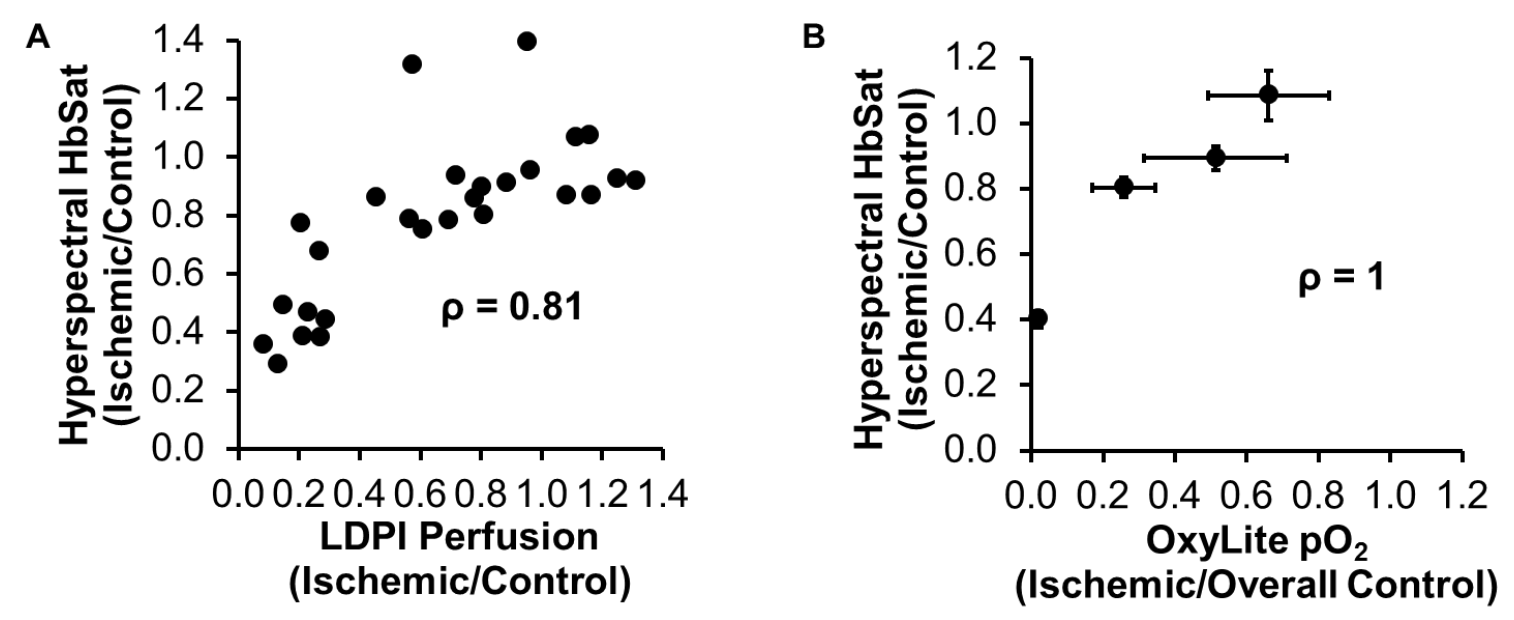

Figure 2.9: Hemoglobin oxygen saturation correlates with perfusion and tissue oxygenation measurements. (A) Hemoglobin saturation correlates with LDPI perfusion data with a Spearman's rank correlation coefficient of $0.81(p<0.001)$. (B) Hemoglobin saturation ratios correlate with OxyLite $\mathrm{pO}_{2}$ ratios with a Spearman's rank correlation coefficient of 1 and $p<0.1$. Data from days 20 (OxyLite) and 21 (HbSat) were compared for the final time point for correlation.

Comparison of the data for individual animals shows that in addition to correlating with current methods of evaluating the hind limb ischemia model, hyperspectral imaging measurements are less variable between animals than LDPI and OxyLite tissue 
oxygenation measurements (Fig. 2.10). Hemoglobin saturation ratio data for $\mathrm{n}=7$ animals over 21 days shows a consistent increasing trend (Fig. 2.10A). LDPI perfusion ratio data for the same $\mathrm{n}=7$ animals over 21 days shows an increasing trend with high inter-animal variation (Fig. 2.10B). OxyLite $\mathrm{pO}_{2}$ ratio data for $\mathrm{n}=5$ animals over 20 days is highly variable and lacks a consistent trend (Fig. 2.10C).
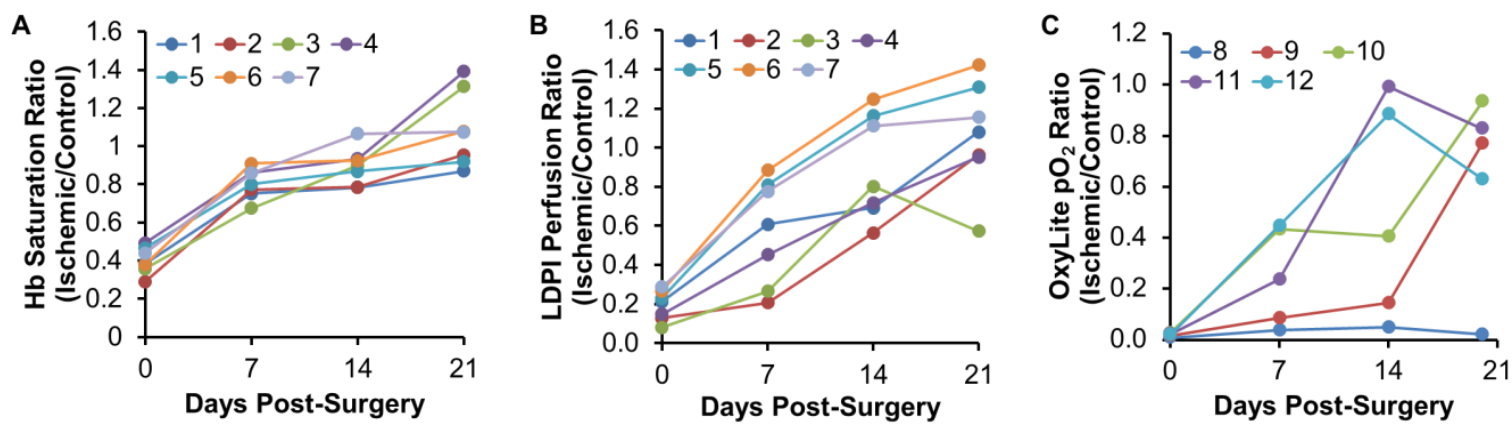

Figure 2.10: Hyperspectral imaging of hemoglobin oxygen saturation is less variable than both LDPI perfusion and OxyLite $\mathrm{pO}_{2}$ measurements in this model. (A) Hemoglobin saturation ratio data for $\mathrm{n}=7$ animals over 21 days shows a consistent increasing trend. (B) LDPI perfusion ratio data for $\mathrm{n}=7$ animals over 21 days shows an increasing trend with high inter-animal variation. (C) OxyLite $\mathrm{pO}_{2}$ ratio data for $\mathrm{n}=5$ animals over 20 days is highly variable and lacks a consistent trend.

Doppler optical coherence tomography imaging of morphology and blood flow

Both morphological and functional images of the hind limb vasculature were acquired non-invasively through the skin with Doppler OCT. The OCT technique provides quantitative structure-function information intravitally using a single instrument rather than the combination of semi-quantitative and post-mortem methods that are currently used to study the hind limb ischemia model.

Two dimensional B-scans oriented perpendicular to the direction of flow in the femoral artery and vein show that flow in these vessels is completely obstructed in the 
ischemic limb immediately after surgery. Representative color-coded Doppler images at day 0 clearly illustrate the presence of flow in the femoral artery (red) and vein (blue) in the control limb (Fig. 2.11A), while there is no Doppler signal from these vessels in the ischemic limb (Fig. 2.11B). Since the vessels turned dark blue after ligation and were visible by eye through the skin, it was known that the vessels were present at the location where the B-scans were acquired in the ischemic limb. By day 7, flow in the femoral artery and vein was being restored in the ischemic limb (Fig. 2.11C), although the magnitude of this response was not consistent across all animals. Quantification of the Bscans in terms of the vascular index as described in Methods resulted in a decrease in the Doppler signal in the ischemic limb that persisted throughout the time course (Fig. 2.11D). A Wilcoxon Signed-Rank test between the control value and the ischemic value at each time point results in a one-sided p-value of 0.0625 , the lowest possible p-value for $\mathrm{n}=4$ animals. The Doppler signal in the femoral artery and vein of the ischemic limb does not correlate well with hemoglobin oxygen saturation or perfusion measurements, indicating that other vessels are compensating for the lack of flow in these major vessels, thus restoring blood flow distally in the footpad. 
A

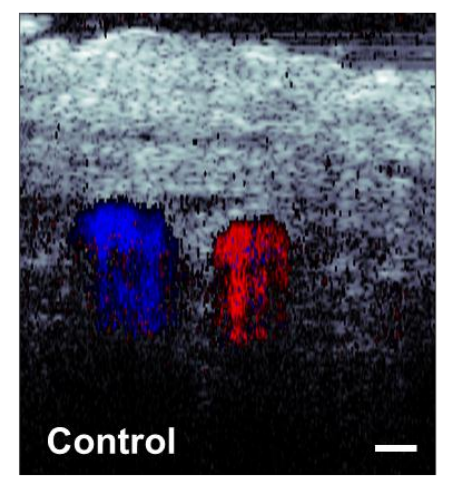

D

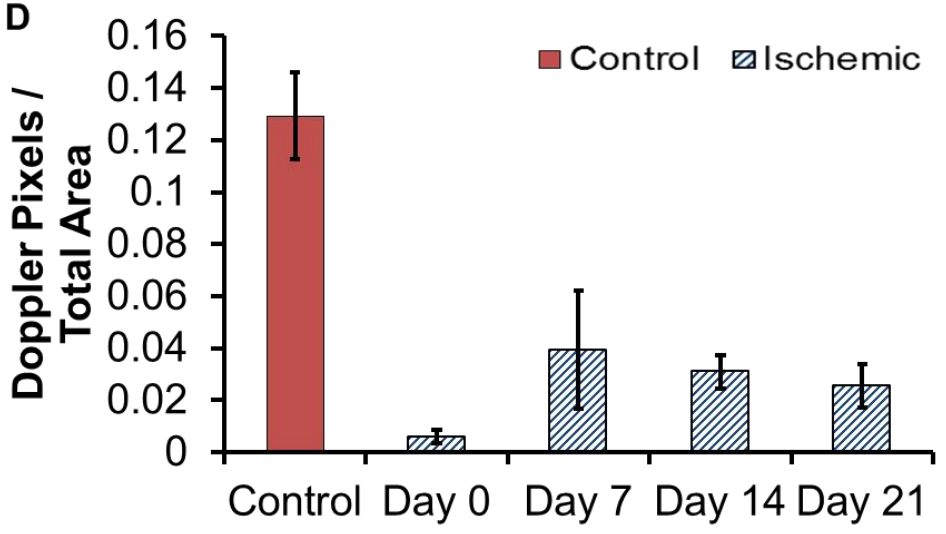

B

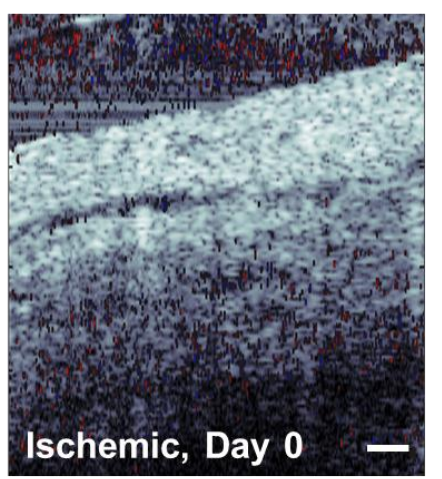

C

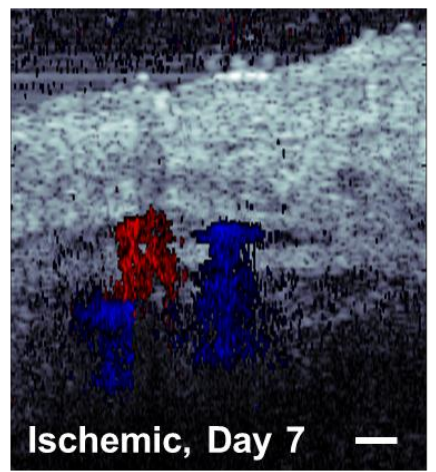

Figure 2.11: Doppler OCT reveals changes in flow through the femoral artery and vein in the ischemic limb after surgery. Representative Doppler OCT B-scans showing the presence or absence of flow in the femoral artery (red) and vein (blue) from the control (A) and ischemic (B) limbs, respectively, at day 0. (C) The Doppler signal in these vessels increases by day 7 in the ischemic limb in one animal. (D) Quantification of the Doppler data shows reduction in flow in the femoral artery region distal to the vessel ligation point. Data presented as mean \pm SEM with $n=4$ animals. Scale bars: $100 \mu \mathrm{m}$.

Maximum intensity projections of Doppler volume data sets from the adductor muscle region of the hind limb revealed changes in flow signal and morphology in the collateral arteries over time (Fig. 2.12). Representative images at day 0 from the control (Fig. 2.12A) and ischemic (Fig. 2.12B) limbs show relatively small vessels with flow appearing to be diverted through a branching vessel in the ischemic limb. A representative volume projection from the adductor at day 7 shows the enlargement and increase in flow in a collateral vessel with the corkscrew morphology that is typical of these remodeling arteries (Fig. 2.12C). At day 21, there is an increase in the number of 
branches coming from a collateral vessel in the adductor muscle region of the ischemic limb indicating that sustained remodeling has occurred in response to the occlusion of the femoral artery and vein (Fig. 2.12D).
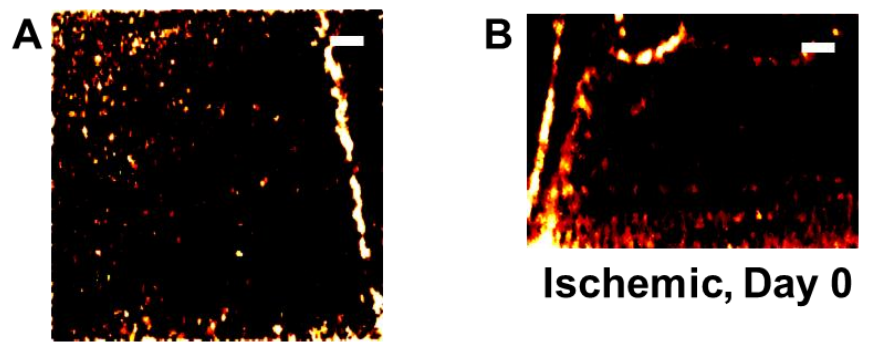

Control, Day 0

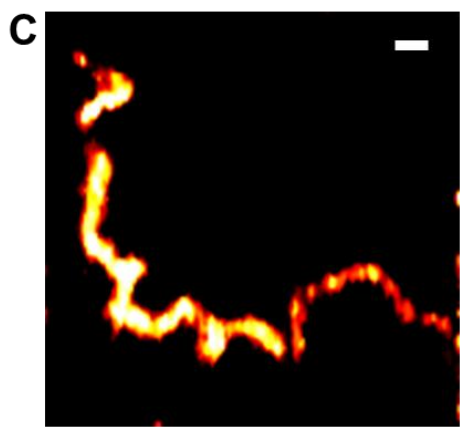

Ischemic, Day 7

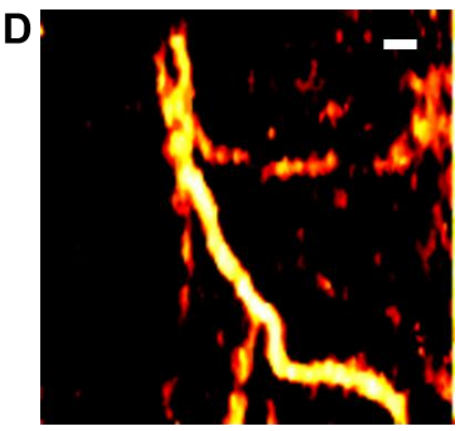

Ischemic, Day 21

Figure 2.12: Representative maximum intensity projections of Doppler volume data sets from the adductor muscle region of the hind limb. (A) Representative image from a contralateral limb shows a small vessel without the corkscrew characteristic of collateral vessels. (B) In the ischemic limb immediately after surgery, flow is being diverted through a branched vessel. (C) By day 7, both flow and size have increased in a collateral vessel with the expected corkscrew morphology that has been described in previous studies [cite]. (D) At day 21, there is increased collateral vessel signal and remodeling in the ischemic limb as shown by the increase in branching points in this vessel. Scale bars: $200 \mu \mathrm{m}$. 


\section{Discussion}

This study was designed to test the feasibility of applying non-invasive, quantitative optical imaging techniques to the mouse hind limb ischemia model of peripheral arterial disease. Quantification of hemoglobin oxygen saturation with hyperspectral imaging was shown to be sensitive to changes in the oxygenation of distal tissue in the ischemic hind limb over a time course of 21 days. This quantitative measurement correlated well with two standard parameters used to track recovery: perfusion ratio and tissue $\mathrm{pO}_{2}$. Additionally, changes in blood flow in the femoral artery and vein distal to the site of vessel occlusion were monitored non-invasively with Doppler OCT. However, recovery of blood flow immediately distal to the ligation site was variable between animals and did not return to control levels, suggesting that a more distal location along the femoral artery may be more suitable for observing vascular recovery with OCT in future studies. Doppler OCT volumes revealed changes in collateral vessel morphology consistent with post-mortem analyses in previous studies $(23,25)$. Doppler OCT enabled acquisition of both functional and morphological data under a single dose of anesthesia with a single instrument.

\section{$\underline{\text { Limitations of standard techniques for hind limb ischemia studies }}$}

LDPI perfusion ratios, $\mathrm{pO}_{2}$ measurements, and post-mortem techniques such as histology, angiography and micro-CT have long been used to study the vascular response in the mouse hind limb ischemia model $(18,23,25,27)$, These methods have enabled valuable insights into the processes of angiogenesis and arteriogenesis, but they are 
limited by a lack of intravital, quantitative data and the need for multiple cohorts of mice for longitudinal studies that rely on post-mortem analyses for quantitative endpoints. While the LDPI perfusion ratio provides a non-invasive, functional endpoint, it is semiquantitative, superficial, and does not provide any information about morphological changes in vasculature. Measurements of $\mathrm{pO}_{2}$ are more quantitative and can be achieved with methods other than the fiber optic sensor-based system used in this study, but even the noninvasive electron paramagnetic resonance (EPR) oximetry method requires injection of an exogenous material (27). The OxyLite sensor measurements acquired in this study were highly variable due to the small sampling volume and decreased sensitivity at normal $\mathrm{pO}_{2}$ levels, but control values were within the range measured by Matsumoto et al. in the femoral muscle with EPR (51). This variability in measurements of normal $\mathrm{pO}_{2}$ makes comparisons more difficult and requires a greater number of mice to establish a control value. Additionally, the animals that underwent $\mathrm{pO}_{2}$ measurements had to be excluded from the hemoglobin saturation and LDPI data sets after it was discovered that the needle-guided insertion of the probe invoked a local expansion of the cutaneous vessels of the footpad, causing changes in hemoglobin saturation and perfusion measurements unrelated to the hind limb ischemia surgery. Post-mortem methods used in hind limb ischemia studies, such as histology, angiography and micro-CT, provide high resolution data for quantifying morphological endpoints, but individual animals cannot be tracked longitudinally and increased numbers of mice must be used to reduce the effects of inter-animal variability that exist even within inbred strains (28). 


\section{$\underline{\text { Hemoglobin oxygen saturation in hind limb ischemia }}$}

The use of hyperspectral imaging to quantify hemoglobin oxygen saturation in the hind limb ischemia model was validated in this study and shows promise as an intravital endpoint for preclinical testing of new therapeutic treatments for PAD. Hyperspectral imaging is non-invasive, and an absolute value can be quantified for a functional measure of recovery in the ischemic footpad over time. Recovery from hypoxia is arguably a more meaningful endpoint than cutaneous blood flow measurements acquired with LDPI, and hyperspectral imaging data are less variable than single point measurements of $\mathrm{pO}_{2}($ Fig. 2.10). Our system acquires a 2-D image of hemoglobin saturation in the entire footpad (Fig. 2.7A), which is an improvement over previous studies that obtained hemoglobin saturation measurements with probe based-systems for optical spectroscopy $(19,25,27)$. Prior to a recent study by Mesquita et al. that used probe-based diffuse optical spectroscopy (19), hemoglobin saturation in hind limb ischemia studies was expressed only as a ratio of the ischemic to control limb rather than an absolute value. The absolute values we measured in the control and ischemic limb are similar to those reported by Mequita et al., although some differences exist in the recovery time course due to differences in the depth and anatomical location of the probed tissue and the severity of the ischemia as determined by the vessel ligation sites. Additionally, while we observed significant improvements in hemoglobin saturation in the ischemic limb between time points, their animals did not experience significant improvements over four weeks which highlights the differences due to surgical procedures.

In addition to reporting the absolute hemoglobin oxygen saturation in the footpad where the most severe ischemia is expected to occur, we were interested in the trends in 
the relative amounts of oxyhemoglobin and deoxyhemoglobin that contribute to the changes in saturation over time. We observed that oxyhemoglobin is significantly decreased and deoxyhemoglobin is significantly increased in the ischemic footpad immediately after surgery as expected (Fig. 2.8). However, oxyhemoglobin levels in the ischemic footpad return to control levels by day 7 and surpassed the control levels at day 21, while deoxyhemoglobin remains elevated through day 14 and a significant decrease in the ischemic to control ratio between time points does not occur until day 21 . The increased oxyhemoglobin content in the ischemic footpad at day 21 supports the hypothesis that smaller vessels have remodeled and increased in number to compensate for the surgically induced obstruction of the large femoral artery. The longer time required for deoxyhemoglobin to return to control levels in the ischemic footpad suggests that tissue demand for and consumption of oxygen is still exceeding the capacity of the remodeling vasculature to deliver oxygenated blood. As perfusion increases and more oxygenated blood is available in the ischemic limb at day 21 , consumption of oxygen no longer outpaces supply. It should be noted that total hemoglobin in the control limb is not constant over time (Fig. 2.8D). This is possibly due to the effects of post-surgery analgesia and decreased activity levels in the mice on circulation. For this reason we did not compare absolute values in the ischemic limb between time points; rather, the ischemic values were first normalized to the control values at each time point.

Previous studies that observed hemoglobin oxygen saturation in the mouse hind limb ischemia model have not discussed the contributing trends in oxyhemoglobin and deoxyhemoglobin. However, clinical studies measuring hemoglobin saturation for human PAD and diabetes patients have recently begun to seek insight into these parameters. 
Chin et al. tested a commercial hyperspectral imaging system to evaluate this technology for assessing PAD in humans (52) and found that there were no trends in oxyhemoglobin that correlated with other measures of PAD such as the ankle-brachial index and Doppler waveforms. Instead, they observed that deoxyhemoglobin content was reduced in the plantar angiosomes affected by PAD and that deoxyhemoglobin decreased with increasing severity of disease. In contrast, other studies of hyperspectral technology report decreases in oxyhemoglobin in diseased and ischemic states such as diabetic foot ulcers (32). Wounds require greater oxygen delivery and extraction in order to heal, and if the vasculature is incapable of meeting the oxygen supply requirements a decrease in oxyhemoglobin at the site would occur as expected. A possible explanation for the contradicting trends observed in these different models is that human PAD is a chronic process which may increase skin blood flow in dependent positions while impairing oxygen extraction (52). The trends observed in this mouse hind limb ischemia study agree more closely with the diabetic wound case and with studies of hemodynamics in acute ischemic events. Nagaoka et al. compressed the brachial artery of a healthy human subject while hemodynamics were measured in the middle finger for one minute (53). Kim et al. measured hemodynamic changes in rat leg muscles during tourniquet-induced ischemia followed by reperfusion (54). In both studies, oxyhemoglobin content was decreased and deoxyhemoglobin content was increased upon induction of ischemia. These trends indicate that the mouse hind limb ischemia model more closely resembles acute ischemic events than chronic human PAD, at least in the early time period after surgery when tissue demand for oxygen exceeds supply. This new insight into the 
hemodynamics of mouse hind limb ischemia should be considered when novel therapeutic treatments for PAD are tested in this preclinical model.

\section{OCT imaging of vessel function and morphology}

The feasibility of obtaining both functional and morphological data in the mouse hind limb noninvasively through the skin with OCT has been successfully demonstrated in this study. Doppler OCT can provide a quantitative measure of blood flow at the resolution level of individual vessels as well as volumetric images of collateral vessel morphology while imaging through the skin without contrast agents or invasive procedures. Here, we detected significant differences in femoral artery blood flow in the ischemic limb relative to the contralateral limb after surgery (Fig. 2.11). Although blood flow was not restored in the femoral artery and vein immediately distal to the ligation site in all of the animals, the functional recovery observed in the footpad (hemoglobin oxygen saturation and perfusion) indicates that more distal locations along the femoral artery may be better regions of interest for future OCT studies. The collateral vessels that compensate for the loss of flow in the proximal femoral artery likely reconnect to and supply the femoral artery at a more distal location than where the OCT scans were collected in this work. In addition to femoral blood flow as a promising functional measure of recovery, we observed changes in collateral vessel morphology (Fig. 2.12) consistent with the tortuous, corkscrew morphology detected in previous studies that used post-mortem techniques to study the phenomenon of arteriogenesis in this model $(23,25$, 27). The ability to visualize these morphological changes intravitally and track individual animals over time is a significant addition to the tools available to researchers working to 
understand the physiological mechanisms of arteriogenesis in basic research as well as those testing novel therapeutic treatments intended to induce collateral vessel formation.

Recently, feasibility of obtaining depth-resolved microvascular images in skeletal muscle with ultrahigh sensitive optical microangiography, an extension of OCT, was demonstrated by Jia et al. (41). In this study, tissue perfusion in the exposed gastrocnemius muscle (via skin incision) was assessed over time following a single ligation of the femoral artery. They observed pronounced reduction in blood supply within one hour of ligation, followed by collateral perfusion at two days and significant perfusion restoration at one week post-ligation. The detection of newly developed collateral vessels and apparent increases in arteriole blood flow at one week agree with the present study in which tortuous, enlarged collateral vessels were observed at the one week time point (Fig. 2.12C). The differences between the perfusion response observed by Jia et al. and that observed in the present study during the early time points are likely due to the differences in the surgical procedure (single ligation versus double ligation and excision of vessel segment) and in the anatomical locations imaged. We selected the adductor muscle as the region of interest to detect collateral vessel remodeling based on previous work demonstrating the diversion of blood flow to the collateral arteries in this muscle (55).

The OCT techniques employed in this study provide insight into both restoration of flow in major vessels (the femoral artery and vein) distal to the ligation sites and changes in vessel function and morphology in the compensatory collateral circulation. The restoration of flow in the distal femoral artery and vein indicates that flow is successfully redirected through collateral arteries that later reconnect to and reperfuse the 
major vessels (Fig. 2.11). These data, along with the functional recovery demonstrated by hyperspectral imaging, also suggest that the collateral arteries undergo remodeling to the extent that they are able to supply blood to the distal tissues at the level of pre-ligation blood flow. The tortuosity of the vessels imaged in the adductor muscle supports the hypothesis that we are in fact detecting arteriogenesis, the process that is thought to be more important than angiogenesis in preventing morbidity and mortality due to arterial obstructive diseases (56). The changes in shear stress which induce arteriogenesis are expected to occur in the pre-existing collateral arterioles in the thigh near the ligation site, while angiogenesis is likely occurring in the distal muscles where hypoxia is more pronounced $(25,50,56)$. Thus, the imaging location in the adductor muscle of the thigh is especially relevant to the study of arteriogenesis in this model. The ability to observe the arteriogenesis process longitudinally through the skin is a unique advantage over current techniques used in this model, because artifacts associated with exposure through skin incisions and post-mortem fixation of tissue and injection of contrast agents can be avoided.

\section{Conclusions and perspectives}

While hyperspectral imaging and quantification of hemoglobin oxygen saturation has several advantages, including the ability to study recovery from ischemia in a quantitative manner within the same animal over time, it is not without limitations. The resolution of our hyperspectral system does not currently allow us to identify individual vessels for co-registration of vessel morphology and function data. However, when hyperspectral imaging is used in combination with OCT techniques we are able to 
acquire complementary morphology and flow data which fills the gaps in acquiring a complete picture of hind limb recovery. Additionally, hemoglobin oxygen saturation data alone does not replace the valuable insights to be gained from high resolution, morphological data provided by techniques such as post-mortem micro-CT (23). Again, we can acquire anatomic data non-invasively with OCT techniques to complete the data set needed for a robust analysis of arteriogenesis and recovery from hypoxia.

Hyperspectral imaging of hemoglobin oxygen saturation and Doppler OCT imaging of vessel morphology and blood flow have significant advantages over commonly used techniques for evaluating vascular recovery in the mouse hind limb ischemia model. The use of multiple intravital methods, such as hyperspectral imaging and OCT, allows for correlations to be made between microvessel morphology and function. This is important because an increase in vessel density or remodeling does not necessarily indicate improved function and oxygen delivery to the ischemic tissue and vice versa. Previous studies have found that certain factors can confound these conclusions, such as the proliferation of unstable and immature vessels in a mouse model of diabetic PAD (57). Thus it is important to obtain a complete picture of changes in the microvasculature over time. The combination of hyperspectral imaging and optical coherence tomography has potential to be a powerful addition to the cardiovascular researcher's toolbox since it is both non-invasive and quantitative. 


\section{CHAPTER 3}

\section{CONCLUSION AND FUTURE DIRECTIONS}

Morphological changes and functional recovery in the hind limb vasculature were studied in the mouse hind limb ischemia model of peripheral arterial disease. Quantitative, intravital and non-invasive optical imaging techniques were applied to obtain new insights into the dynamics of recovery in this model and address a significant, unmet need for an improved methodological toolbox for angiogenesis researchers. Hemoglobin oxygen saturation was quantified in areas distal to the site of vessel occlusion using hyperspectral imaging, and changes in vessel morphology and flow were visualized and quantified using optical coherence tomography. Hemoglobin oxygen saturation correlated with traditional perfusion measurements, validating this quantitative endpoint for use in this preclinical model. Additionally, OCT provided previously unattainable visualization of hind limb vasculature in a given mouse over time without the need for contrast agents, in contrast to the post-mortem techniques of micro-CT and histology. These results suggest that hyperspectral imaging and OCT may be further

developed as tools for studying the mechanisms of vascular recovery in this model and evaluating new therapeutic treatments in preclinical studies. 


\section{Quantitative Endpoints for Optical Imaging Methods}

The results presented in Chapter 2 indicate that hyperspectral imaging and optical coherence tomography are valid techniques for studying the hind limb ischemia model. However, the full power of OCT to provide robust, quantitative endpoints has not yet been utilized. Examples of quantitative endpoints that could be derived from OCT data are summarized in Table 3.1. Feasibility of extracting absolute velocity from the hind limb vasculature in mice has been demonstrated in a pilot experiment (Fig. 3.1). In this experiment, the absolute blood velocity along the center line of the vessel was computed from the phase shift measured by Doppler OCT using the relation described by Davis et al. (58) which corrects the data for the angle of incidence and fits the flow profile to a second order polynomial. From the velocity profile in a vessel of interest, the blood flow rate and vessel wall shear rate can be calculated as defined in Table 3.1. In addition to extracting quantitative endpoints from Doppler OCT images, software can be developed to automatically segment the vessels in three dimensions using filtering techniques applied to the speckle variance data. This segmentation would facilitate quantification of the vascular volume fraction and connectivity endpoints. Finally, the structure-function relationships in the vasculature can be visualized by overlaying the Doppler data onto the

corresponding morphological image which allows for visualization of blood flow dynamics in the context of vessel structure. 
Table 3.1. Endpoints to be derived from speckle variance and Doppler OCT data.

\begin{tabular}{|ll|}
\hline $\begin{array}{l}\text { Endpoint } \\
\text { fraction }\end{array}$ & $\begin{array}{l}\text { Definition } \\
\text { Volume of all vessels / total tissue volume in region of } \\
\text { interest }\end{array}$ \\
\hline Connectivity & $\begin{array}{l}\text { Maximal number of branches that can be broken within a } \\
\text { structure before it is divided into two separate parts }\end{array}$ \\
\hline Blood flow rate & $\begin{array}{l}1 / 2 \mathrm{~V}_{\max } \pi \mathrm{r}^{2}, \text { where } \mathrm{V}_{\max } \text { is the maximum blood velocity } \\
\text { and } \mathrm{r} \text { is the vessel radius }\end{array}$ \\
\hline Vessel wall shear rate & $\begin{array}{l}\text { Derivative of the 2nd order polynomial fit to the velocity } \\
\text { flow profile at the vessel boundary }\end{array}$ \\
\hline Vascular index & $\begin{array}{l}\text { Doppler-positive pixel count integrated over the volume of } \\
\text { interest }\end{array}$ \\
\hline
\end{tabular}

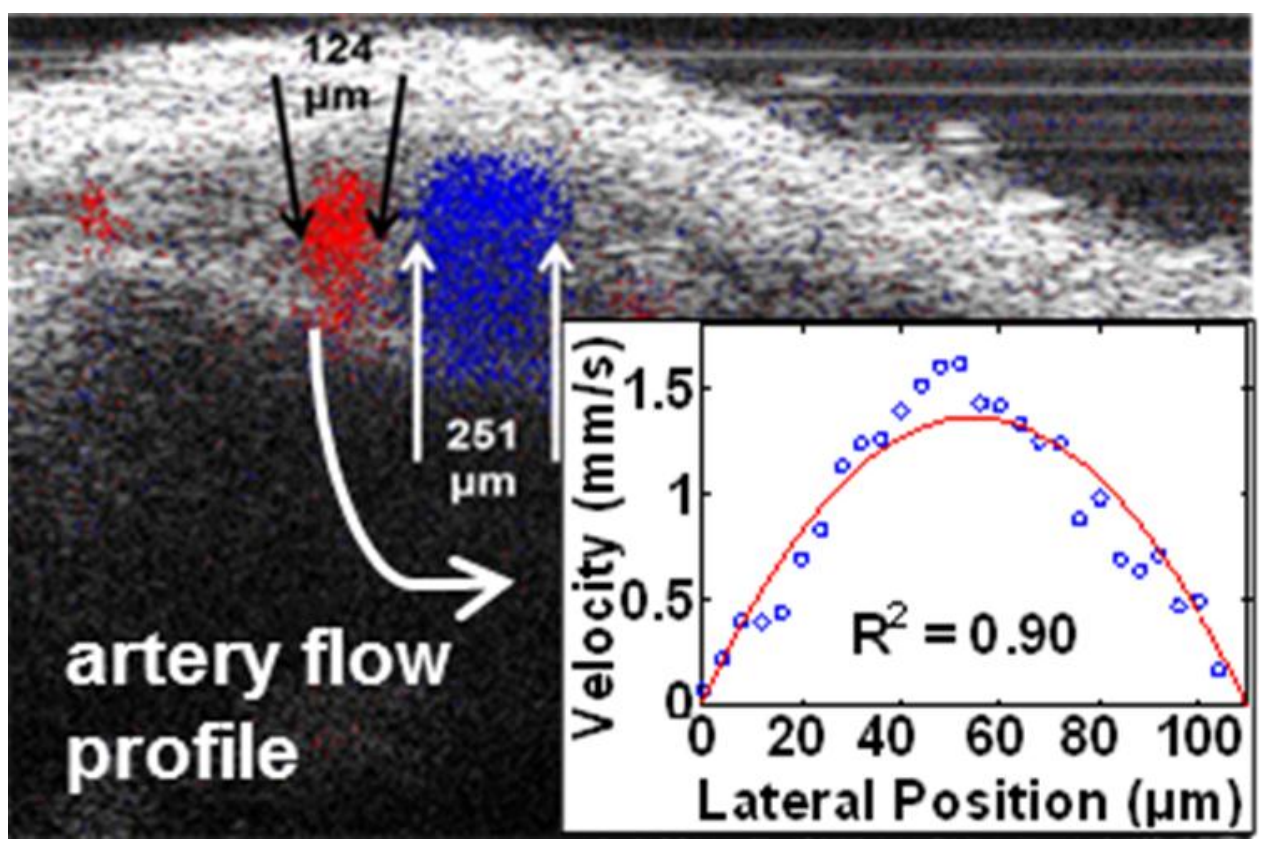

Figure 3.1: Doppler OCT of femoral artery and vein in a control hind limb. A Doppler OCT B-scan was acquired through the skin of the control hind limb of a mouse. Blue and red colored pixels indicate blood flow in opposite directions. The inset is a plot of the velocity flow profile in the femoral artery with a second order polynomial curve fit.

With these quantitative endpoints, the OCT techniques can be more robustly compared to the established methods of studying the hind limb ischemia model including micro-CT and LDPI. Additionally, new insight into the physiological processes of arteriogenesis 
and angiogenesis may be gained through measurement of previously unattainable parameters such as the shear rate on the vessel wall.

\section{Future Directions}

After image processing techniques and extraction of quantitative endpoints from OCT and hyperspectral data have been optimized, we will seek to validate the sensitivity of the optical imaging methods with a known proangiogenic perturbation applied to the hind limb ischemia model. A growth factor such as VEGF or FGF will be administered in protein form encapsulated in biodegradable nanoparticles for sustained delivery as described in previous studies (22). VEGF has been shown to accelerate neovascularization in the mouse hind limb ischemia model (22), and FGF has been shown to induce as much if not greater collateral vessel formation compared to VEGF (59). Comparison between mice receiving treatment and control mice that do not receive a growth factor will provide a means to test the sensitivity of the hyperspectral and OCT techniques for detecting the differences in treatment versus control outcomes. Sensitivity to treatment effects is critical to using these techniques to evaluate novel therapeutic treatments in the future.

Another future direction of interest is a study of the hind limb ischemia model in diabetic mice. As described in Chapter 1, diabetes mellitus (DM) significantly increases risk and severity of peripheral arterial disease. Diabetes is linked to impaired collateral vessel formation and reduced treatment response $(7,60)$, indicating a need for studies that more clearly elucidate the differences between revascularization in diabetic and non- 
diabetic PAD. It is believed that shear stress is a key stimulus for arteriogenesis, but to date there is no experimental evidence of how this mechanism is dysfunctional in DM (61-63).

The streptozotocin model of DM has been used extensively in preclinical research to study the pathways that lead to insulitis and $\beta$ cell death (20). The mouse hind limb ischemia model (18) has been used to study postnatal blood vessel formation and plays a vital role in testing new therapeutic angiogenesis approaches as described in Chapter 1. Recently, these models have been combined in an effort to develop better treatments for DM-PAD patients $(64,65)$. If the diabetes phenotype is characterized with more informative and quantitative techniques such as hyperspectral imaging and OCT, it may lend insights that will ultimately lead to the development of more effective therapeutic treatments for diabetic PAD patients. 


\section{REFERENCES}

1. Roger VL, Go AS, Lloyd-Jones DM, Benjamin EJ, Berry JD, Borden WB, et al. Heart Disease and Stroke Statistics--2012 Update: A Report From the American Heart Association. Circulation.125(1):e2-e220.

2. Leeper NJ, Kullo IJ, Cooke JP. Genetics of peripheral artery disease. Circulation.125(25):3220-8.

3. Peripheral arterial disease in people with diabetes. Diabetes Care. 2003;26(12):3333-41.

4. Weitz JI, Byrne J, Clagett GP, Farkouh ME, Porter JM, Sackett DL, et al. Diagnosis and treatment of chronic arterial insufficiency of the lower extremities: a critical review. Circulation. 1996;94(11):3026-49.

5. Petznick AM, Shubrook JH. Treatment of specific macrovascular beds in patients with diabetes mellitus. Osteopath Med Prim Care. 2010;4:5. PMCID: 2925360.

6. Papanas N, Maltezos E. Advances in treating the ischaemic diabetic foot. Curr Vasc Pharmacol. 2008;6(1):23-8.

7. Khurana R, Simons M, Martin JF, Zachary IC. Role of angiogenesis in cardiovascular disease: a critical appraisal. Circulation. 2005;112(12):1813-24.

8. Carmelli D, Fabsitz RR, Swan GE, Reed T, Miller B, Wolf PA. Contribution of genetic and environmental influences to ankle-brachial blood pressure index in the NHLBI Twin Study. National Heart, Lung, and Blood Institute. Am J Epidemiol. 2000;151(5):452-8.

9. Kullo IJ, Turner ST, Kardia SL, Mosley TH, Jr., Boerwinkle E, de Andrade M. A genome-wide linkage scan for ankle-brachial index in African American and nonHispanic white subjects participating in the GENOA study. Atherosclerosis. 2006;187(2):433-8.

10. Murabito JM, Guo CY, Fox CS, D'Agostino RB. Heritability of the ankle-brachial index: the Framingham Offspring study. Am J Epidemiol. 2006;164(10):963-8.

11. Hirsch AT, Criqui MH, Treat-Jacobson D, Regensteiner JG, Creager MA, Olin JW, et al. Peripheral arterial disease detection, awareness, and treatment in primary care. JAMA. 2001;286(11):1317-24.

12. Hankey GJ, Norman PE, Eikelboom JW. Medical treatment of peripheral arterial disease. JAMA. 2006;295(5):547-53. 
13. Morishita R. Perspective in progress of cardiovascular gene therapy. J Pharmacol Sci. 2004;95(1):1-8.

14. Baumgartner I, Pieczek A, Manor O, Blair R, Kearney M, Walsh K, et al. Constitutive expression of phVEGF165 after intramuscular gene transfer promotes collateral vessel development in patients with critical limb ischemia. Circulation. 1998;97(12):1114-23.

15. Comerota AJ, Throm RC, Miller KA, Henry T, Chronos N, Laird J, et al. Naked plasmid DNA encoding fibroblast growth factor type 1 for the treatment of end-stage unreconstructible lower extremity ischemia: preliminary results of a phase I trial. J Vasc Surg. 2002;35(5):930-6.

16. Taniyama Y, Morishita R, Aoki M, Nakagami H, Yamamoto K, Yamazaki K, et al. Therapeutic angiogenesis induced by human hepatocyte growth factor gene in rat and rabbit hindlimb ischemia models: preclinical study for treatment of peripheral arterial disease. Gene Ther. 2001;8(3):181-9.

17. Tongers J, Roncalli JG, Losordo DW. Therapeutic angiogenesis for critical limb ischemia: microvascular therapies coming of age. Circulation. 2008;118(1):9-16.

18. Couffinhal T, Silver M, Zheng LP, Kearney M, Witzenbichler B, Isner JM. Mouse model of angiogenesis. Am J Pathol. 1998;152(6):1667-79. PMCID: 1858441.

19. Mesquita RC, Skuli N, Kim MN, Liang J, Schenkel S, Majmundar AJ, et al. Hemodynamic and metabolic diffuse optical monitoring in a mouse model of hindlimb ischemia. Biomed Opt Express.1(4):1173-87. PMCID: 3018079.

20. Rees DA, Alcolado JC. Animal models of diabetes mellitus. Diabet Med. 2005;22(4):359-70.

21. Rivard A, Silver M, Chen D, Kearney M, Magner M, Annex B, et al. Rescue of diabetes-related impairment of angiogenesis by intramuscular gene therapy with adenoVEGF. Am J Pathol. 1999;154(2):355-63. PMCID: 1850015.

22. Golub JS, Kim YT, Duvall CL, Bellamkonda RV, Gupta D, Lin AS, et al. Sustained VEGF delivery via PLGA nanoparticles promotes vascular growth. Am J Physiol Heart Circ Physiol. 2010;298(6):H1959-65. PMCID: 2886627.

23. Duvall CL, Taylor WR, Weiss D, Guldberg RE. Quantitative microcomputed tomography analysis of collateral vessel development after ischemic injury. Am J Physiol Heart Circ Physiol. 2004;287(1):H302-10.

24. Duvall CL, Weiss D, Robinson ST, Alameddine FM, Guldberg RE, Taylor WR. The role of osteopontin in recovery from hind limb ischemia. Arterioscler Thromb Vasc Biol. 2008;28(2):290-5. 
25. Scholz D, Ziegelhoeffer T, Helisch A, Wagner S, Friedrich C, Podzuweit T, et al. Contribution of arteriogenesis and angiogenesis to postocclusive hindlimb perfusion in mice. J Mol Cell Cardiol. 2002;34(7):775-87.

26. Palmer GM, Viola RJ, Schroeder T, Yarmolenko PS, Dewhirst MW, Ramanujam N. Quantitative diffuse reflectance and fluorescence spectroscopy: tool to monitor tumor physiology in vivo. J Biomed Opt. 2009;14(2):024010. PMCID: 2724679.

27. Helisch A, Wagner S, Khan N, Drinane M, Wolfram S, Heil M, et al. Impact of mouse strain differences in innate hindlimb collateral vasculature. Arterioscler Thromb Vasc Biol. 2006;26(3):520-6.

28. Zbinden S, Clavijo LC, Kantor B, Morsli H, Cortes GA, Andrews JA, et al. Interanimal variability in preexisting collaterals is a major factor determining outcome in experimental angiogenesis trials. Am J Physiol Heart Circ Physiol. 2007;292(4):H1891-7.

29. Sorg BS, Moeller BJ, Donovan O, Cao Y, Dewhirst MW. Hyperspectral imaging of hemoglobin saturation in tumor microvasculature and tumor hypoxia development. $\mathbf{J}$ Biomed Opt. 2005;10(4):44004.

30. Palmer GM, Fontanella AN, Shan S, Hanna G, Zhang G, Fraser CL, et al. In vivo optical molecular imaging and analysis in mice using dorsal window chamber models applied to hypoxia, vasculature and fluorescent reporters. Nat Protoc. 2011;6(9):1355-66.

31. Shonat RD, Wachman ES, Niu W, Koretsky AP, Farkas DL. Near-simultaneous hemoglobin saturation and oxygen tension maps in mouse brain using an AOTF microscope. Biophys J. 1997;73(3):1223-31. PMCID: 1181022.

32. Khaodhiar L, Dinh T, Schomacker KT, Panasyuk SV, Freeman JE, Lew R, et al. The use of medical hyperspectral technology to evaluate microcirculatory changes in diabetic foot ulcers and to predict clinical outcomes. Diabetes Care. 2007;30(4):903-10.

33. Skala MC, Fontanella A, Hendargo H, Dewhirst MW, Izatt JA. Combined hyperspectral and spectral domain optical coherence tomography microscope for noninvasive hemodynamic imaging. Opt Lett. 2009;34(3):289-91. PMCID: 2715151.

34. Gebhart SC, Thompson RC, Mahadevan-Jansen A. Liquid-crystal tunable filter spectral imaging for brain tumor demarcation. Appl Opt. 2007;46(10):1896-910.

35. Jacques SL. Skin Optics. Oregon Medical Laser Center News; 1998 [updated 1998; cited]; Available from: omlc.ogi.edu/news/jan98/skinoptics.html.

36. Mariampillai A, Standish BA, Moriyama EH, Khurana M, Munce NR, Leung MK, et al. Speckle variance detection of microvasculature using swept-source optical coherence tomography. Opt Lett. 2008;33(13):1530-2. 
37. Izatt JA, Kulkarni MD, Yazdanfar S, Barton JK, Welch AJ. In vivo bidirectional color Doppler flow imaging of picoliter blood volumes using optical coherence tomography. Opt Lett. 1997;22(18):1439-41.

38. Yazdanfar S, Kulkarni M, Izatt J. High resolution imaging of in vivo cardiac dynamics using color Doppler optical coherence tomography. Opt Express. 1997;1(13):424-31.

39. Costa RA, Skaf M, Melo LA, Jr., Calucci D, Cardillo JA, Castro JC, et al. Retinal assessment using optical coherence tomography. Prog Retin Eye Res. 2006;25(3):325-53.

40. Ben-Dor I, Mahmoudi M, Pichard AD, Satler LF, Waksman R. Optical coherence tomography: a new imaging modality for plaque characterization and stent implantation. J Interv Cardiol.24(2):184-92.

41. Jia Y, Qin J, Zhi Z, Wang RK. Ultrahigh sensitive optical microangiography reveals depth-resolved microcirculation and its longitudinal response to prolonged ischemic event within skeletal muscles in mice. J Biomed Opt. 2011;16(8):086004. PMCID: 3162619.

42. Yang VXD, Gordon ML, Mok A, Zhao YH, Chen ZP, Cobbold RSC, et al. Improved phase-resolved optical Doppler tomography using the Kasai velocity estimator and histogram segmentation. Optics Communications. 2002;208(4-6):209-14.

43. Standish BA, Yang VXD, Munce NR, Song LMWK, Gardiner G, Lin A, et al. Doppler optical coherence tomography monitoring of microvascular tissue response during photodynamic therapy in an animal model of Barrett's esophagus. Gastrointestinal Endoscopy. 2007;66(2):326-33.

44. Jones WS, Annex BH. Growth factors for therapeutic angiogenesis in peripheral arterial disease. Curr Opin Cardiol. 2007;22(5):458-63.

45. Srinivasan VJ, Jiang JY, Yaseen MA, Radhakrishnan H, Wu W, Barry S, et al. Rapid volumetric angiography of cortical microvasculature with optical coherence tomography. Opt Lett.35(1):43-5. PMCID: 2912612.

46. Skala MC, Crow MJ, Wax A, Izatt JA. Photothermal optical coherence tomography of epidermal growth factor receptor in live cells using immunotargeted gold nanospheres. Nano Lett. 2008;8(10):3461-7. PMCID: 2574829.

47. Skala MC, Fontanella A, Lan L, Izatt JA, Dewhirst MW. Longitudinal optical imaging of tumor metabolism and hemodynamics. J Biomed Opt. 2010;15(1):011112. PMCID: 2816992. 
48. Drexler W, Fujimoto JG. State-of-the-art retinal optical coherence tomography. Prog Retin Eye Res. 2008;27(1):45-88.

49. Pierce MC, Strasswimmer J, Park BH, Cense B, de Boer JF. Advances in optical coherence tomography imaging for dermatology. J Invest Dermatol. 2004;123(3):458-63.

50. Chalothorn D, Faber JE. Strain-dependent variation in collateral circulatory function in mouse hindlimb. Physiol Genomics. 2010;42(3):469-79. PMCID: 2929883.

51. Matsumoto A, Matsumoto S, Sowers AL, Koscielniak JW, Trigg NJ, Kuppusamy $\mathrm{P}$, et al. Absolute oxygen tension $(\mathrm{pO}(2))$ in murine fatty and muscle tissue as determined by EPR. Magn Reson Med. 2005;54(6):1530-5.

52. Chin JA, Wang EC, Kibbe MR. Evaluation of hyperspectral technology for assessing the presence and severity of peripheral artery disease. J Vasc Surg. 2011;54(6):1679-88.

53. Nagaoka T, Eikje NS, Nakamura A, Aizawa K, Kiyohara Y, Ichikawa F, et al. Inspection of skin hemodynamics with hyperspectral camera. Conf Proc IEEE Eng Med Biol Soc. 2007;2007:3357-61.

54. Kim JG, Lee J, Roe J, Tromberg BJ, Brenner M, Walters TJ. Hemodynamic changes in rat leg muscles during tourniquet-induced ischemia-reperfusion injury observed by near-infrared spectroscopy. Physiol Meas. 2009;30(7):529-40. PMCID: 2868521.

55. Limbourg A, Korff T, Napp LC, Schaper W, Drexler H, Limbourg FP. Evaluation of postnatal arteriogenesis and angiogenesis in a mouse model of hind-limb ischemia. Nat Protoc. 2009;4(12):1737-46.

56. Schirmer SH, van Nooijen FC, Piek JJ, van Royen N. Stimulation of collateral artery growth: travelling further down the road to clinical application. Heart. 2009;95(3):191-7.

57. Landazuri N, Joseph G, Guldberg RE, Taylor WR. Growth and Regression of Vasculature in Healthy and Diabetic Mice after Hind Limb Ischemia. Am J Physiol Regul Integr Comp Physiol. 2012;In press.

58. Davis AM, Rothenberg FG, Shepherd N, Izatt JA. In vivo spectral domain optical coherence tomography volumetric imaging and spectral Doppler velocimetry of early stage embryonic chicken heart development. J Opt Soc Am A Opt Image Sci Vis. 2008;25(12):3134-43.

59. Wafai R, Tudor EM, Angus JA, Wright CE. Vascular effects of FGF-2 and VEGF-B in rabbits with bilateral hind limb ischemia. J Vasc Res. 2009;46(1):45-54. 
60. Lederman RJ, Mendelsohn FO, Anderson RD, Saucedo JF, Tenaglia AN, Hermiller JB, et al. Therapeutic angiogenesis with recombinant fibroblast growth factor-2 for intermittent claudication (the TRAFFIC study): a randomised trial. Lancet. 2002;359(9323):2053-8.

61. Schaper W, Ito WD. Molecular mechanisms of coronary collateral vessel growth. Circ Res. 1996;79(5):911-9.

62. Papadaki M, Eskin SG, Ruef J, Runge MS, McIntire LV. Fluid shear stress as a regulator of gene expression in vascular cells: possible correlations with diabetic abnormalities. Diabetes Res Clin Pract. 1999;45(2-3):89-99.

63. Waltenberger J. Impaired collateral vessel development in diabetes: potential cellular mechanisms and therapeutic implications. Cardiovasc Res. 2001;49(3):554-60.

64. Bir SC, Esaki J, Marui A, Sakaguchi H, Kevil CG, Ikeda T, et al. Therapeutic treatment with sustained-release platelet-rich plasma restores blood perfusion by augmenting ischemia-induced angiogenesis and arteriogenesis in diabetic mice. $\mathrm{J}$ Vasc Res. 2011;48(3):195-205.

65. Raiter A, Bechor Z, Kleiman M, Leshem-Lev D, Battler A, Hardy B. Angiogenic peptides improve blood flow and promote capillary growth in a diabetic and ischaemic mouse model. Eur J Vasc Endovasc Surg. 2011;40(3):381-8. 